\title{
Nonflatness and totality
}

\author{
BASIL A. KARÁDAIS \\ Mathematisches Institut, Ludwig-Maximilians-Universität München, Munich 80333, Germany \\ Email: karadais@math.lmu.de
}

Received 21 October 2016; revised 10 December 2017

\begin{abstract}
We interpret finite types as domains over nonflat inductive base types in order to bring out the finitary core that seems to be inherent in the concept of totality. We prove a strong version of the Kreisel density theorem by providing a total compact element as a witness, a result that we cannot hope to have if we work with flat base types. To this end, we develop tools that deal adequately with possibly inconsistent finite sets of information. The classical density theorem is reestablished via a 'finite density theorem,' and corollaries are obtained, among them Berger's separation property.
\end{abstract}

\section{Introduction}

In the area of denotational semantics of functional programming, it is standard to view data types as countably based Scott domains in the tradition that started with Scott's and Ershov's independent work in the late sixties and early seventies. More particularly, we may view these domains through their representations as Scott information systems, where programs are representatives of typed terms $x: \rho$ with denotations being ideals in appropriate information systems, that is, consistent and deductively closed sets of tokens $a \in x$; ideals are approximated by finite sets $U \subseteq x$, their so called formal neighbourhoods.

A crucial choice in our setting is to work with nonflat rather than flat domains for the base types. These arise when we model base type partiality not as an extra pseudotoken, but as an extra nullary pseudoconstructor, which participates in the formation of further tokens, and therefore leads to varying degrees of partiality. For example, while the flat natural numbers $\{\perp, 0, \mathrm{~S} 0, \mathrm{SS} 0, \ldots\}$ feature just the bottom element for partiality, the nonflat natural numbers (also called lazy natural numbers) $\{\perp, 0, \mathrm{~S} \perp, \mathrm{S} 0, \mathrm{SS} \perp, \mathrm{SS} 0, \ldots\}$ feature several partial elements, like $\perp, \mathrm{S} \perp, \mathrm{SS} \perp$ and so on; elements that do not involve $\perp$, like $0, \mathrm{SO}$ or SSO, are called total. A basic advantage of this feature compared to flat base types is that we obtain injectivity and disjoint ranges for the constructors.

More generally, base-type nonflatness yields domains which are in a certain sense both richer, in that they contain more tokens, and tidier, in that they are finitely branching. Such domains seem to accommodate arguments that a flat setting cannot afford, and this paper intends to give one nontrivial example of this kind: an explicitly finitary approach to the Kreisel density theorem, a key result in the theory of higher-type computability.

Density was first stated and proved by Kreisel (1959), and in different terms by Kleene (1959). Building on the work of Ershov (1975a,b, 1977), Berger (1990, 1993) generalised and established density within domain theory, drawing as a corollary that it holds for the hierarchy of the partial continuous functionals over all finite types, and thus recovering 
the Kleene-Kreisel continuous functionals as equivalence classes of the abstractly total elements in the hierarchy. Schwichtenberg and collaborators have already carried Berger's argument from the top (abstract domains with totality) down to the bottom (concrete Scott information systems induced by algebra constructors) numerous times in the past, starting with Schwichtenberg (1996) and following up with Schwichtenberg (2007), Huber (2010), Huber et al. (2010), Schwichtenberg et al. (2012). The present work is related to these top-down approaches, but attacks the problem in the opposite, bottom-up manner: we do not merely adapt previous domain-theoretic proofs to the setting of approximations, but rather work our way up, from approximations to ideals.

Let us recall the content of the Kreisel density theorem. We work with finite types, that is, with base types like $\mathbb{N}$ and $\mathbb{B}$ for naturals and booleans, respectively, and then with arrow types above them. We capture the concept of termination by a totality predicate $G:^{\dagger}$ at base types $l$, an ideal $x$ is total if it contains a total token; at type $\rho \rightarrow \sigma$, an ideal $f$ is total when it preserves totality, that is, when

$$
\underset{x: \rho}{\forall}\left(G_{\rho}(x) \rightarrow G_{\sigma}(f x)\right),
$$

where $b \in f x$ for a token $b \in \operatorname{Tok}_{\sigma}$ if and only if $\langle U, b\rangle \in f$, for some formal neighbourhood $U \in \operatorname{Con}_{\rho}$ with $U \subseteq x$. The density property for a type, the latter being understood as a space governed by the Scott topology, alleviates the omnipresent partiality by ensuring that every open set in the space nurtures total points, in other words, that total points are dense in the space. We formulate this here by saying that $\rho$ is dense when

$$
\underset{U \in \operatorname{Con}_{\rho}}{\forall} \underset{x: \rho}{\exists}\left(G_{\rho}(x) \wedge U \subseteq x\right) .
$$

The Kreisel density theorem says that every type is dense.

Let us look a bit closer at the statement (D). We are given a neighbourhood $U$, morally a compact element, comprising finite information, and we are supposed to come up with an ideal $x$ as a witness, which may in principle be an infinite set of tokens. It is reasonable to suspect that the element of infinity in $x$ must be inessential as far as an actual process of 'totalisation' of $U$ is concerned - whatever this process might be - and that there is nothing inherently infinitary about it, since totalising $U$ should depend on its finite information, and not on the fact that the resulting ideal may be infinite. Can we then devise a totalisation process which will feature an explicitly finitary core, that is,

can we provide a witness for density which will be obviously finitary?

We can easily see that in general, we cannot do this if we interpret our base types by flat domains: At type $\mathbb{N} \rightarrow \mathbb{N}$, consider the very simple compact given intuitively by $\{0 \mapsto \perp\}$; extending it, say, to $\{0 \mapsto 0\}$ is not making it total, since it cannot respond (with a total value) to any total input different than 0 ; if we want to extend it to a total element,

\footnotetext{
$\dagger$ The letter 'g' - which probably derives from the English word general (for generally defined), and perhaps is influenced by the German word gesamt (complete, total, whole) - was used in this context already by Ershov (1975a, 1977). We adopt this notation here to designate totality in order to avoid confusion with other terms beginning with ' $t$ ' in our text.
} 
we must account for every possible total input except 0 , which inevitably leads to an infinite set, say to the set $\{0 \mapsto 0\} \cup\left\{\mathrm{S}^{m} 0 \mapsto \mathrm{S} 0 \mid m>0\right\}$. This totalisation process consists of two steps, namely totalising the output on input zero from $\perp$ to 0 , and setting all other outputs to be S0; nevertheless, the witness can only be presented as an infinite set. On the other hand, if we had interpreted the natural numbers by their nonflat domain, then we could have extended the compact $\{0 \mapsto \perp\}$ to the compact $\{0 \mapsto 0, \mathrm{~S} \perp \mapsto \mathrm{S} 0\}$, which would have sufficed, since the information $\mathrm{S} \perp$ is enough to accommodate (later we will say 'accept') all total numbers different than zero.

Our strategy can be summarised as follows. Step 1: define an appropriate notion of 'total neighbourhood.' Step 2: establish a 'finite density theorem,' that is, that every neighbourhood extends to such a total neighbourhood. Step 3: show that a total neighbourhood extends to a total ideal in a straightforward way.

We begin in Section 2 with a necessary preamble on domains over nonflat base types represented by information systems. In Section 3, we prepare for Step 1. As we can already see in the example that we gave above, one thing we have to do in order to 'totalise' a neighbourhood is to appropriately extend the set of its inputs; this finite set is seldom consistent, and in order to argue rigorously about it, we must first develop a general understanding of such sets and their intricacies. This section delays the exposition of our argument a bit, but, beside gathering necessary definitions and facts, it will hopefully help familiarise the reader with these intricacies. Alternatively, the reader could skip to the next section and come back when the need arises. In Section 4, we perform Steps 1 and 2: we define finite totality and prove finite density with Theorem 4.7; moreover, we characterise finite totality in a noninductive way in Theorem 4.9. Section 5 is about Step 3: with Theorem 5.10, we show how to obtain the classical Kreisel density theorem from the finite density theorem; in addition, we list some direct consequences, among them Berger's 'separation property' in Proposition 5.14. We end in Section 6 with comments on the literature and future work.

\section{Nonflat domains via coherent information systems}

We concentrate on a type system supporting arrow types over inductive base types. $\$$ We use $\xi$ as a dummy-type variable. Write $\vec{\rho} \rightarrow \sigma$ to mean $\rho_{1} \rightarrow \cdots \rightarrow \rho_{r} \rightarrow \sigma$ for some $r \geqslant 0$ associated to the right; in case $r=0$, the vector is empty.

- For every vector $\vec{\xi}$ of length $r$, the expression $\vec{\xi} \rightarrow \xi$ is a constructor type (of arity $r)$.

- If $\kappa_{1}, \ldots, \kappa_{k}$ are constructor types for $k>0$ and one of them nullary, then $\mu_{\xi}\left(\kappa_{1}, \ldots, \kappa_{k}\right)$ is a type. We think of such types as inductively defined base types or algebras, generated by constructors $C_{l}$ corresponding to $\kappa_{l}$, for $l=1, \ldots, k$.

- If $\rho, \sigma$ are types, then $\rho \rightarrow \sigma$ is a type; these are the usual higher types.

$¥$ In this section, we omit proofs and details, for which the reader may consult Schwichtenberg et al. (2012, Part 3) and Stoltenberg-Hansen et al. (1994, Part I). In relation to the former, in particular, note that we will be working within the nonparametric and finitary fragment of the system. 
Note that constructor types only serve to build base types, and are not themselves admitted as types. Examples of base types are

- the unit type $\mathbb{U}:=\mu_{\xi}(\xi)$ with a single nullary constructor,

- the type of boolean values $\mathbb{B}:=\mu_{\xi}(\xi, \xi)$, with constructors for the truth $t t: \mathbb{B}$ and the falsity ff : $\mathbb{B}$,

- the type of natural numbers $\mathbb{N}:=\mu_{\xi}(\xi, \xi \rightarrow \xi)$, with constructors for the zero $0: \mathbb{N}$ and the successor $\mathrm{S}: \mathbb{N} \rightarrow \mathbb{N}$,

— the type of (extended) derivations $\mathbb{D}:=\mu_{\xi}(\xi, \xi, \xi \rightarrow \xi, \xi \rightarrow \xi \rightarrow \xi)$, with constructors for an axiom $0: \mathbb{D}$, another axiom $1: \mathbb{D}$, a one-premise rule $S: \mathbb{D} \rightarrow \mathbb{D}$, and a two-premise rule $\mathrm{B}: \mathbb{D} \rightarrow \mathbb{D} \rightarrow \mathbb{D}$ (this algebra is simple yet nontrivial enough to provide us with examples as we go along).

We will write $\imath$ to denote an arbitrary base type and $\rho, \sigma$ to denote arbitrary types in general.

A (Scott) information system (Scott 1982; Winskel and Larsen 1984) is a triple (Tok, Con, $\vdash$ ), where Tok is an inhabited countable set of tokens, Con is a collection of finite sets of tokens, which we call consistent sets or (formal) neighbourhoods, and $\vdash$ is a subset of Con $\times$ Tok, the entailment. These are subject to the axioms

$$
\begin{aligned}
& \{a\} \in \text { Con, } \\
& U \subseteq V \wedge V \in \text { Con } \rightarrow U \in \text { Con, } \\
& U \in \text { Con } \wedge a \in U \rightarrow U \vdash a, \\
& U \vdash V \wedge V \vdash c \rightarrow U \vdash c, \\
& U \in \operatorname{Con} \wedge U \vdash b \rightarrow U \cup\{b\} \in \text { Con, }
\end{aligned}
$$

where $U \vdash V$ stands for $U \vdash b$ for all $b \in V$. From the latter follows vacuously that $U \vdash \varnothing$ for all $U$, while $\varnothing \in$ Con follows from the first two axioms. We may refer to the fifth axiom as propagation (of consistency through entailment); note that this axiom may be equivalently expressed as

$$
U \vdash V \rightarrow U \cup V \in \text { Con. }
$$

For finite sets of tokens $\Gamma$, which are not necessarily consistent, we write Fin, so Con $\subseteq$ Fin. An information system is called coherent when in addition to the above it satisfies

$$
\underset{a, a^{\prime} \in U}{\forall}\left\{a, a^{\prime}\right\} \in \text { Con } \rightarrow U \in \text { Con, }
$$

for all $U \in$ Fin. By the coherence and the second axiom above, it follows that the consistency of a token set is equivalent to the consistency of its pairs. Drawing on this property, we often write $a \asymp b$ for $\{a, b\} \in$ Con, and even $U \asymp V$ for $U \cup V \in$ Con (which is also often written $U \uparrow V$ ). In the following, we work exclusively with coherent systems, even if we do not mention it explicitly.

Given two coherent information systems $\rho$ and $\sigma$, we form their function space $\rho \rightarrow \sigma:$ define its tokens by $\langle U, b\rangle \in$ Tok if $U \in \operatorname{Con}_{\rho}$ and $b \in \operatorname{Tok}_{\sigma}$, its consistency by $\langle U, b\rangle \asymp$ 
$\left\langle U^{\prime}, b^{\prime}\right\rangle$ if $U \asymp_{\rho} U^{\prime}$ implies $b{ }_{\sigma} b^{\prime}$, and its entailment by $W \vdash\langle U, b\rangle$ if $W U \vdash_{\sigma} b$, where

$$
b \in W U:=\underset{U^{\prime} \in \operatorname{Con}_{\rho}}{\exists}\left(\left\langle U^{\prime}, b\right\rangle \in W \wedge U \vdash_{\rho} U^{\prime}\right) .
$$

The last operation is called neighbourhood application. We will revisit it in some depth in Section 3.3, where we will also show that it is monotone in both arguments, that is, that $U \vdash U^{\prime}$ implies $W U \vdash W U^{\prime}$ and that $W \vdash W^{\prime}$ implies $W U \vdash W U^{\prime}$ for all appropriate $U, U^{\prime}, W, W^{\prime}$ (Lemma 3.8). For the proof of the following, see Schwichtenberg et al. (2012, Chapter 6).

Fact 2.1. The function space of two coherent systems is itself a coherent information system.

An ideal (or element) of an information system $\rho$ is a possibly infinite token set $x \subseteq$ Tok, such that $U \in$ Con for every $U \subseteq_{f} x$ (consistency), and $U \vdash b$ for some $U \subseteq_{f} x$ implies $b \in x$ (deductive closure). If $x$ is an ideal of $\rho$, we write $x: \rho$ or $x \in \operatorname{Ide}_{\rho}$. Note that in a generic setting built over flat base types, as for example, the one described in StoltenbergHansen et al. (1994), the empty set at every type $\rho$ is an ideal, and plays the role of the bottom element $\perp_{\rho}$.

By a (Scott-Ershov) domain, we mean here a countably based directed complete partial order with a least element, which is additionally algebraic and bounded complete. A domain is coherent (Plotkin 1978), if every set of compacts has a least upper bound exactly when each of its pairs has a least upper bound. Write $b \in \bar{U}$ if and only if $U \vdash b$ (in the generic flat-based setting, we have $\bar{\varnothing}=\varnothing=\perp$ ). The following fact is directly based on the fundamental work of Scott (1982); for the proofs supporting our particular formulation, see Stoltenberg-Hansen et al. (1994, Section 6.1) and Karádais (2016, Theorem 8).

Fact 2.2 (Representation theorem). Let $\rho=\left(\operatorname{Tok}_{\rho}, \operatorname{Con}_{\rho}, \vdash_{\rho}\right)$ be a coherent information system. Then, ( $\left.\operatorname{Ide}_{\rho}, \subseteq, \perp_{\rho}\right)$ is a coherent domain with compacts given by $\left\{\bar{U} \mid U \in \operatorname{Con}_{\rho}\right\}$. Conversely, every coherent domain can be represented by a coherent information system.

An approximable mapping between two information systems $\rho$ and $\sigma$ is a relation $r \subseteq \mathrm{Con}_{\rho} \times \mathrm{Con}_{\sigma}$ that generalises entailment in the following sense: $\langle\varnothing, \varnothing\rangle \in r$; if $\left\langle U, V_{1}\right\rangle,\left\langle U, V_{2}\right\rangle \in r$, then $V_{1} \asymp_{\sigma} V_{2}$ and $\left\langle U, V_{1} \cup V_{2}\right\rangle \in r$; and if $U \vdash_{\rho} U^{\prime},\left\langle U^{\prime}, V^{\prime}\right\rangle \in r$, and $V^{\prime} \vdash_{\sigma} V$, then $\langle U, V\rangle \in r$. One can show (Scott 1982) that there is a bijective correspondence between the approximable mappings from $\rho$ to $\sigma$ and the ideals of the function space $\rho \rightarrow \sigma$, and moreover establish the categorical equivalence between domains with Scott continuous functions and information systems with approximable mappings. The equivalence is preserved if we restrict ourselves to the coherent case on both sides (Karádais 2016).

The Scott topology on $\operatorname{Ide}_{\rho}$ is given by the collection $\left\{\nabla U \mid U \in \operatorname{Con}_{\rho}\right\}$, where $\nabla U$ is the set $\{x: \rho \mid U \subseteq x\}$ of all ideals above $U$. A set $\mathcal{U} \subseteq \operatorname{Ide}_{\rho}$ of ideals is Scott open when it is closed under supersets (Alexandrov condition) and for every $x \in \mathcal{U}$, there is a $U \subseteq x$ such that $\bar{U} \in \mathcal{U}$ (Scott condition). One can furthermore show that an ideal-mapping $f$ sending ideals from Ide $\operatorname{Ido}_{\rho}$ ideals in $\mathrm{Ide}_{\sigma}$ is Scott continuous when it is monotone and 
satisfies the principle of finite support (also called approximation principle) for all $x: \rho$, that is,

$$
\underset{b \in \operatorname{Tok}_{\sigma}}{\forall}\left(b \in f(x) \rightarrow \underset{U \in \operatorname{Con}_{\rho}}{\exists}(U \subseteq x \wedge b \in f(\bar{U}))\right) .
$$

Finally, it can be shown (see, for example, Schwichtenberg et al. 2012, Section 6.1.3) that the ideals $\operatorname{Ide}_{\rho \rightarrow \sigma}$ and the Scott continuous ideal-mappings $\operatorname{Ide}_{\rho} \rightarrow \operatorname{Ide}_{\sigma}$ are in a bijective correspondence, a fact that justifies the nondiscriminating notation $f: \rho \rightarrow \sigma$.

Now, we proceed to assign an information system to each type. Every higher type is naturally assigned a function space, so it suffices to discuss the information systems for base types, that is, for algebras. Let $\imath$ be an algebra, with at least one nullary constructor if it is to be nontrivial. We add to it an extra nullary pseudoconstructor $*{ }_{l}$ (or just $*$ ) to denote partiality (which we nevertheless agree to not let it appear in the algebra signature). This is a departure from Schwichtenberg et al. (2012), where partiality is treated as a special untyped symbol that is used by all possible algebras; for us, every algebra has its own partiality symbol. We define $\mathrm{Tok}_{l}, \mathrm{Con}_{l}$, and $\vdash_{l}$ inductively.

- If $C$ is an $r$-ary constructor and $a_{i} \in \mathrm{Tok}_{l}$ for $i=1, \ldots, r$, then $C a_{1} \cdots a_{r} \in \mathrm{Tok}_{l} \S^{\S}$ For its head constructor, write $\operatorname{hd}\left(C a_{1} \cdots a_{r}\right)=C$; for its ith component token write $a(i)$, that is, $\left(C a_{1} \cdots a_{r}\right)(i)=a_{i}$ for $i=1, \ldots, r$.

- We have $a \asymp_{l} *$ and $* \asymp_{l} a$ for all $a \in \operatorname{Tok}_{l}$. Furthermore, if $C$ is an $r$-ary constructor and $a_{i} \asymp_{l} b_{i}$ for $i=1, \ldots, r$, then $C a_{1} \cdots a_{r} \asymp_{l} C b_{1} \cdots b_{r}$. Finally, we have $U \in \mathrm{Con}_{l}$ if $a \asymp_{l} a^{\prime}$ for all $a, a^{\prime} \in U$.

- We have $U \vdash_{l} *$ for all $U \in$ Con $_{l}$. Furthermore, if $C$ is an $r$-ary constructor, every $U_{i} \in \mathrm{Con}_{l}$ is inhabited and $U_{i} \vdash_{l} b_{i}$ for $i=1, \ldots, r$, then $U \vdash_{l} C b_{1} \cdots b_{r}$ for all $U \in \mathrm{Con}_{l}$ that are sufficient for $C$ on $U_{1}, \ldots, U_{r}$, in the sense that for each $i=1, \ldots, r$ and each $a_{i} \in U_{i}$ there exists an $a \in U$ such that $\operatorname{hd}(a)=C$ and $a(i)=a_{i}$. Finally, if $U \vdash_{l} b$, then also $U \cup\{*\} \vdash_{l} b$.

Here, we need to be cautious. The definition of $\mathrm{Con}_{l}$ incorporates the coherence property (2), so it follows that $\varnothing \vdash_{l}\{*\}$. This seems to undermine Fact 2.2, since the empty set cannot be an ideal anymore. It is nevertheless straightforward to show that the representation theorem still stands if we reinterpret the bottom ideals as follows:

$$
\begin{aligned}
\perp_{l} & :=\left\{*_{l}\right\}, \\
\perp_{\rho \rightarrow \sigma} & :=\left\{\langle U, b\rangle \mid U \in \operatorname{Con}_{\rho} \wedge b \in \perp_{\sigma}\right\} .
\end{aligned}
$$

It may be worth pointing out that while our situation diverges from the generic flat-based setting and even from settings like the one of Schwichtenberg et al. (2012), in the same time, it resonates the original Scott axioms (Scott 1982): there exists at least one trivial token in every information system, in particular, one at every base type and several at every higher type.

Concerning sufficiency, we note the following: (a) in case $C$ is a proper constructor, $U$ is sufficient for $C$ on $U_{1}, \ldots, U_{r}$ if and only if $U \cup\{*\}$ is, if and only if $U \backslash\{*\}$ is, and

$\S$ Throughout the text, we adopt the polish notation for tokens for typographical convenience. 
(b) we trivially have $U \vdash_{l} C U_{1} \cdots U_{r}$, whenever $U$ is sufficient for $C$ on $U_{1}, \ldots, U_{r}$; the constructor application here is defined by

$$
C U_{1} \cdots U_{r}:=\left\{C a_{1} \cdots a_{r} \mid a_{1} \in U_{1}, \ldots, a_{r} \in U_{r}\right\},
$$

which is consistent if and only if every $U_{i}$ is consistent. Moreover, every neighbourhood $U$ that is nontrivial (meaning, $\{*\} H_{l} U$ ) is indeed equivalent to one of the form $C U_{1} \cdots U_{r}$ : if

$$
U \backslash\{*\}=\left\{C a_{11} \cdots a_{r 1}, \ldots, C a_{1 m} \cdots a_{r m}\right\},
$$

we gather all $i$ th component tokens into a neighbourhood, the ith component neighbourhood $U(i):=\left\{a_{i 1}, \ldots, a_{i m}\right\}$ of $U$, and let $U_{i}:=U(i)$ for every $i=1, \ldots, r$; then, we indeed have $U \sim{ }_{l} C U_{1} \cdots U_{r}$ (where $U \sim V$ abbreviates $U \vdash V \wedge V \vdash U$ ).

The proof of the following is straightforward but tedious.

Fact 2.3. Let $\imath$ be an algebra given by constructors. The triple $\left(\operatorname{Tok}_{l}, \mathrm{Con}_{l}, \vdash_{l}\right)$ is a coherent information system.

\section{Finite sets}

Recall that the first step in our strategy is to decide on a reasonable definition of 'finite totality,' one that will already embody the totalisation mechanism for density on the one hand, and that will be susceptible to a canonical extension to a total ideal on the other. The nature of our study leads us naturally to define the concept by induction over types, which is what we do in Section 4.1. Nevertheless, we will see in Theorem 4.9 that we can arrive at the same concept in an explicit way in terms of 'transitive elements': elements that witness local transitivity within a not necessarily transitive relation - in our case, the consistency relation. To work with such sets of tokens, we need an operation akin to application, defined using consistency rather than entailment. This is how we come to spend some space discussing not necessarily consistent finite sets in some generality, while we postpone the actual definition of total neighbourhoods until Section 4.

\subsection{Entailment and consistency for finite sets}

There is the trivial syntactical reason to look at finite sets in general and not just at the consistent ones: the latter presuppose the former by definition - in particular, the thematisation of finite sets is unavoidable in implementation endeavors like (Huber et al. 2010). But finite sets may play a natural and important role within purely semantical arguments as well - to mention a naive example, think of the subtokens $a_{1}, \ldots, a_{r}$ of a base-type token $a=C a_{1} \cdots a_{r}$. In this subsection, we will hardly cover anything more than what we will need later, with the exception of Lemma 3.2, which we included for the sake of some points in Section 3.3.

As we already mentioned, we write $\operatorname{Fin}_{\rho}$ instead of $\mathcal{P}_{f}\left(\operatorname{Tok}_{\rho}\right)$, so $\Gamma \in \operatorname{Fin}_{\rho}$ means that $\Gamma$ is a finite set of tokens, not necessarily consistent. If $\Theta=\left\{\left\langle U_{j}, b_{j}\right\rangle \mid j=1, \ldots, l\right\} \in \operatorname{Fin}_{\rho \rightarrow \sigma}$, write $L(\Theta)$ for $\bigcup_{j} U_{j} \in \operatorname{Fin}_{\rho}$ (notice that this is a flattening), and $R(\Theta)$ for $\bigcup_{j}\left\{b_{j}\right\} \in \operatorname{Fin}_{\sigma}$. 
Furthermore, if $U \in \operatorname{Con}_{\rho}$ and $\Delta \in \operatorname{Fin}_{\sigma}$, write $\langle U, \Delta\rangle$ for $\{\langle U, b\rangle \mid b \in \Delta\} \in \operatorname{Fin}_{\rho \rightarrow \sigma}$ (note that $\left.\left\langle U, \varnothing_{\sigma}\right\rangle=\varnothing_{\rho \rightarrow \sigma}\right)$.

Lemma 3.1. Let $\Theta, \Theta^{\prime} \in \operatorname{Fin}_{\rho \rightarrow \sigma}$. We have $L\left(\Theta \cup \Theta^{\prime}\right)=L(\Theta) \cup L\left(\Theta^{\prime}\right)$ and $R(\Theta \cup$ $\left.\Theta^{\prime}\right)=R(\Theta) \cup R\left(\Theta^{\prime}\right)$. Furthermore, if $\Theta \subseteq \Theta^{\prime}$, then we have $L(\Theta) \subseteq L\left(\Theta^{\prime}\right)$ as well as $R(\Theta) \subseteq R\left(\Theta^{\prime}\right)$.

A neighbourhood in $\Gamma \in$ Fin $_{\rho}$ is a subset $U \subseteq \Gamma$, which happens to be consistent; write $U \in \operatorname{Con}_{\Gamma}$. The empty set and the singletons of $\Gamma$ are always in $\operatorname{Con}_{\Gamma}$. Say that $\Gamma$ entails $\Gamma^{\prime}$ (as a finite set), and write $\Gamma \vdash_{\rho}^{F} \Gamma^{\prime}$, when

$$
\underset{U^{\prime} \in \operatorname{Con}_{\Gamma^{\prime}}}{\forall} \underset{U \in \operatorname{Con}_{\Gamma}}{\exists} U \vdash_{\rho} U^{\prime} .
$$

This is a generalisation of the notion $U \vdash_{\rho} U^{\prime}$ for neighbourhoods. A simpler, but less helpful generalisation is ' $\Gamma \vdash_{\rho} \Gamma^{\prime}$ if and only if for every $a^{\prime} \in \Gamma^{\prime}$ there is some $U \in \operatorname{Con}_{\Gamma}$ such that $U \vdash_{\rho} a^{\prime \prime}$; if $\Gamma \vdash_{\rho}^{F} \Gamma^{\prime}$, then also $\Gamma \vdash_{\rho} \Gamma^{\prime}$. Contrary to the case of consistent sets, although $\Gamma \vdash_{\rho}^{F} \Gamma^{\prime}$ implies $\Gamma \vdash_{\rho}^{F} a$ for all $a \in \Gamma^{\prime}$, the converse is not true in general. For example, $\{\mathrm{B} 00, \mathrm{~B} 11\} \vdash_{\mathbb{D}}^{F} \mathrm{~B} 0 *$ and $\{\mathrm{B} 00, \mathrm{~B} 11\} \vdash_{\mathbb{D}}^{F} \mathrm{~B} * 1$, but $\{\mathrm{B} 00, \mathrm{~B} 11\} \nvdash_{\mathbb{D}}^{F}\{\mathrm{~B} 0 *, \mathrm{~B} * 1\}$.

Similarly, say that $\Gamma$ and $\Gamma^{\prime}$ are consistent (as finite sets), and write $\Gamma \asymp_{\rho}^{F} \Gamma^{\prime}$, when

$$
\underset{U \in \operatorname{Con}_{\Gamma}}{\forall} \underset{U^{\prime} \in \operatorname{Con}_{\Gamma}^{\prime}}{\forall} U \asymp \rho U^{\prime} .
$$

Again, this is a generalisation of consistency between neighbourhoods that proves more useful for not necessarily consistent finite sets than the simpler notion ' $\Gamma \asymp_{\rho} \Gamma^{\prime}$ if and only if $\left\{a, a^{\prime}\right\} \in \operatorname{Con}_{\rho}$ for all $a \in \Gamma$ and $a^{\prime} \in \Gamma^{\prime \prime}$ (which we may nevertheless occasionally use); if $\Gamma \asymp_{\rho}^{F} \Gamma^{\prime}$, then $\Gamma \asymp_{\rho} \Gamma^{\prime}$. Note that in the case of $\asymp_{\rho}^{F}$, we generally do not have reflexivity; in fact, we trivially have $\Gamma \asymp_{\rho}^{F} \Gamma$ if and only if $\Gamma \in \operatorname{Con}_{\rho}$. An example of consistency between inconsistent finite sets is $\{B 0 *, B 1 *\} \asymp_{\mathbb{D}}^{F}\{\mathrm{~B} * 0, \mathrm{~B} * 1\}$.

Reflexivity of consistency is the only property that the triple $\left(\operatorname{Fin}_{\rho}, \asymp_{\rho}^{F}, \vdash_{\rho}^{F}\right.$ ) lacks in order to constitute a Scott information system.

Lemma 3.2. The entailment between finite sets is reflexive and transitive and the consistency between finite sets is symmetric and propagates through entailment, that is,

1. $\forall_{\Gamma \in \text { Fin }} \Gamma \vdash^{F} \Gamma$,

2. $\forall_{\Gamma, \Delta, \Theta \in \operatorname{Fin}}\left(\Gamma \vdash^{F} \Delta \wedge \Delta \vdash^{F} \Theta \rightarrow \Gamma \vdash^{F} \Theta\right)$,

3. $\forall_{\Gamma, \Delta \in \text { Fin }}\left(\Gamma \asymp^{F} \Delta \rightarrow \Delta \asymp^{F} \Gamma\right)$,

4. $\forall_{\Gamma, \Delta, \Theta \in \operatorname{Fin}}\left(\Gamma \asymp^{F} \Delta \wedge \Delta \vdash^{F} \Theta \rightarrow \Gamma \asymp^{F} \Theta\right)$.

Proof. We just show the propagation property. Let $\Gamma, \Delta, \Theta \in$ Fin be such that $\Gamma \asymp F \Delta$ and $\Delta \vdash^{F} \Theta$. Consider $U \in \operatorname{Con}_{\Gamma}$ and $W \in \operatorname{Con}_{\Theta}$; by the assumptions, there exists a $V \in \mathrm{Con}_{\Delta}$ with $V \vdash W$ and $U \asymp V$; by propagation on Con (1), we get $U \asymp W$.

\subsection{Maximal and transitive neighbourhoods}

Think of some finite set $\Gamma$ of type $\rho$, and suppose that we wish to assign $\sigma$-values $b_{i}$ to neighbourhoods $U_{i}$ of $\operatorname{Con}_{\Gamma}$ (for some $i \in I$ ) in a way that the finite set $\left\{\left\langle U_{i}, b_{i}\right\rangle \mid i \in I\right\}$ at type $\rho \rightarrow \sigma$ will be consistent. Some reflection show that it suffices to pair the 'maximal' 
neighbourhoods of $\Gamma$ with the given values of $\sigma$, but we can actually do better than that: we can relax the requirement of maximality by requiring instead that we assign the given arbitrary values already to those neighbourhoods which are, so to speak, maximal enough or 'almost maximal,' in the sense that they are below exactly one maximal in $\Gamma$; these are exactly the 'transitive neighbourhoods' in $\Gamma$.

Call $U \in \operatorname{Con}_{\Gamma}$ a maximal neighbourhood in $\Gamma$, and write $U \in \operatorname{Con}_{\Gamma}^{\max }$, when it is maximal with respect to the entailment relation, that is, when

$$
\underset{U^{\prime} \in \operatorname{Con}_{\Gamma}}{\forall}\left(U^{\prime} \vdash_{\rho} U \rightarrow U \vdash_{\rho} U^{\prime}\right) .
$$

Call $U \in \operatorname{Con}_{\Gamma}$ (consistency) transitive in $\Gamma$, and write $U \in \operatorname{Con}_{\Gamma}^{\mathrm{ctr}}$, when it satisfies the property

$$
\underset{U_{1}, U_{2} \in \operatorname{Con}_{\Gamma}}{\forall}\left(U_{1} \asymp_{\rho} U \asymp_{\rho} U_{2} \rightarrow U_{1} \asymp_{\rho} U_{2}\right) .
$$

We can reformulate this by introducing the notation $\widetilde{U}$ for the consistency closure of $U$, that is, for the set $\left\{a \in \operatorname{Tok}_{\rho} \mid U \asymp_{\rho} a\right\}$ (it is clear that, while it encompasses the deductive closure, the consistency closure of a neighbourhood is not in general an ideal, because consistency may fail); then $U$ is transitive in $\Gamma$ when $\widetilde{U} \cap \Gamma \in \operatorname{Con}_{\rho}$.

More generally, call $U \in \operatorname{Con}_{\rho}$ transitive for $\Gamma$ or just $\Gamma$-transitive (in $\rho$ ), and write $U \in \operatorname{Con}_{\rho \mid \Gamma}^{\mathrm{ctr}}$, if, again, $U_{1} \asymp_{\rho} U \asymp_{\rho} U_{2}$ implies $U_{1} \asymp{ }_{\rho} U_{2}$ for all $U_{1}, U_{2} \in \operatorname{Con}_{\Gamma}$; obviously, $\operatorname{Con}_{\Gamma}^{\mathrm{ctr}} \subseteq \operatorname{Con}_{\rho \mid \Gamma}^{\mathrm{ctr}}$.

It is clear that every maximal in a finite set is also transitive in it. It is also immediate that consistency between neighbourhoods, restricted to $\operatorname{Con}_{\Gamma}^{\mathrm{ctr}}$ (but not to $\operatorname{Con}_{\rho \mid \Gamma}^{\mathrm{ctr}}$ !), becomes an equivalence relation. Still trivially, but importantly, we have the following.

Lemma 3.3 (Upward closedness of transitivity). Let $\rho$ be a type and $\Gamma \in \operatorname{Fin}_{\rho}$. For any $U, U^{\prime} \in \operatorname{Con}_{\rho}$, if $U \in \operatorname{Con}_{\rho \mid \Gamma}^{\mathrm{ctr}}$ and $U^{\prime} \vdash_{\rho} U$, then $U^{\prime} \in \operatorname{Con}_{\rho \mid \Gamma}^{\mathrm{ctr}}$.

Proof. Let $U_{1}, U_{2} \in \operatorname{Con}_{\Gamma}$ be such that $U_{1} \asymp{ }_{\rho} U^{\prime} \asymp{ }_{\rho} U_{2}$. By propagation (1), we have $U_{1} \asymp{ }_{\rho} U \asymp U_{2}$, so $U_{1} \asymp{ }_{\rho} U_{2}$.

It is often handy to check for extremality (that is, maximality or transitivity) on the level of tokens.

Lemma 3.4 (Extremality through tokens). Let $\rho$ be an arbitrary type, $\Gamma \in \mathrm{Fin}_{\rho}$, and $U \in \operatorname{Con}_{\Gamma}$.

1. We have $U \in \operatorname{Con}_{\Gamma}^{\max }$ if and only if $U \asymp_{\rho} a$ implies $U \vdash_{\rho} a$ for $a \in \Gamma$.

2. We have $U \in \operatorname{Con}_{\rho \mid \Gamma}^{\mathrm{ctr}}$ if and only if $a_{1} \asymp_{\rho} U \asymp_{\rho} a_{2}$ implies $a_{1} \asymp_{\rho} a_{2}$ for $a_{1}, a_{2} \in \Gamma$.

Proof. For 1. From left to right, assume that $U$ is maximal, and let $a \in \Gamma$ be such that $U \asymp_{\rho} a$. Then, $U \cup\{a\} \vdash_{\rho} U$, and by the maximality of $U$, we get $U \sim_{\rho} U \cup\{a\}$, which gives us $U \vdash_{\rho}$ a. For the other way around, let $U^{\prime} \in \operatorname{Con}_{\Gamma}$ be such that $U^{\prime} \vdash_{\rho} U$; then $U \asymp_{\rho} U^{\prime}$, and by the assumption we get that $U \vdash_{\rho} U^{\prime}$, so $U$ is indeed maximal.

For 2. From left to right, assume that $U$ is transitive for $\Gamma$, and let $a_{1}, a_{2} \in \Gamma$ be such that $U \asymp_{\rho} a_{i}$ for both $i$. Then, $U \asymp_{\rho}\left\{a_{i}\right\}$, and by the transitivity of $U$ we get $a_{1} \asymp_{\rho} a_{2}$. For 
the other way around, let $U_{1}, U_{2} \in \operatorname{Con}_{\Gamma}$ be such that $U \asymp_{\rho} U_{i}$, for both $i$ and $a_{i} \in U_{i}$; then $a_{1} \asymp \rho a_{2}$ by the assumption, so $U_{1} \asymp \rho U_{2}$, and $U$ is indeed $\Gamma$-transitive.

The lemma makes the significance of extremality in a finite set quite apparent. In particular, it is good to know that two maximals in a finite set are either equivalent or inconsistent (a fact that we can put even more bluntly like this: if $U$ is maximal and deductively closed in $\Gamma$, then for each $a \in \Gamma$, we have either $a \in U$ or $a \star_{\rho} U$ ).

Lemma 3.5. Let $\rho$ be any type. For all $\Gamma \in \operatorname{Fin}_{\rho}$ and $U \in \operatorname{Con}_{\Gamma}^{\max }$, if $U^{\prime} \in \operatorname{Con}_{\rho}$ is such that $U^{\prime} \vdash_{\rho} U$, then $U^{\prime} \in \operatorname{Con}_{\Gamma \cup U^{\prime}}^{\max }$.

Proof. Let $a \in \Gamma \cup U^{\prime}$ be such that $a \asymp_{\rho} U^{\prime}$; since $U^{\prime} \vdash_{\rho} U$, we have $a \asymp_{\rho} U$. In case $a \notin \Gamma$, we have $a \in U^{\prime}$; in case $a \in \Gamma$, we have $U \vdash_{\rho} a$ by Lemma 3.4 (1); in both the cases, it follows that $U^{\prime} \vdash_{\rho} a$, so $U^{\prime}$ is maximal in $\Gamma \cup U^{\prime}$ by Lemma 3.4 (1).

Lemma 3.6 (Maximal extensions). Let $\rho$ be a type and $\Gamma \in \operatorname{Fin}_{\rho}$.

1. For any $U \in \operatorname{Con}_{\Gamma}$, we have $U \in \operatorname{Con}_{\Gamma}^{\text {ctr }}$ if and only if there is exactly one $\hat{U} \in \operatorname{Con}_{\Gamma}^{\max }$, up to equientailment, such that $\hat{U} \vdash_{\rho} U$.

2. For any $U \in \operatorname{Con}_{\rho}$, we have $U \in \operatorname{Con}_{\rho \mid \Gamma}^{\text {ctr }}$ if and only if, whenever there exist $U_{0} \in \operatorname{Con}_{\Gamma}$ with $U \asymp{ }_{\rho} U_{0}$, there exists a $\hat{U} \in \operatorname{Con}_{\Gamma}^{\max }$ such that $U \asymp_{\rho} U_{0}$ implies $\hat{U} \vdash_{\rho} U_{0}$ for all $U_{0} \in \operatorname{Con}_{\Gamma}$.

Proof. For 1, from left to right, assume that $U \in \operatorname{Con}_{\Gamma}^{\text {ctr }}$ and let $U_{1}, U_{2} \in \operatorname{Con}_{\Gamma}^{\max }$ be such that $U_{i} \vdash_{\rho} U$ for both $i=1,2$. By the propagation of consistency (1), we have $U_{1} \asymp_{\rho} U \asymp_{\rho} U_{2}$; by the assumption, we have $U_{1} \asymp{ }_{\rho} U_{2}$; by the maximality of $U_{1}$ and $U_{2}$, it follows from Lemma 3.4 (1) that $U_{1} \sim_{\rho} U_{2}$.

For the other direction, assume that $U$ is such that any two maximal neighbourhoods in $\Gamma$ that entail it are equivalent, and let $U_{1}, U_{2} \in \operatorname{Con}_{\Gamma}$ be such that $U_{1} \asymp_{\rho} U \asymp_{\rho} U_{2}$. Then, for any two $U_{1}^{m}, U_{2}^{m} \in \operatorname{Con}_{\Gamma}^{\max }$, with $U_{i}^{m} \vdash_{\rho} U \cup U_{i}$, by the assumption, we must have $U_{1}^{m} \sim{ }_{\rho} U_{2}^{m}$; it follows that $U_{1} \asymp{ }_{\rho} U_{2}$, by the propagation of consistency.

For 2, let $U \in \operatorname{Con}_{\rho}$. Assume that $U \in \operatorname{Con}_{\rho \mid \Gamma}^{\text {ctr }}$ and $U \asymp \asymp_{\rho} U_{i}$ for some $U_{i} \in \operatorname{Con}_{\Gamma}$, where $i>0$. Gather all these $U_{i}$ in the neighbourhood $U_{0}:=\bigcup_{i} U_{i}$; we have of course $U_{0} \in \operatorname{Con}_{\Gamma}$. Then, there is at least one maximal $\hat{U} \in \operatorname{Con}_{\Gamma}^{\max }$ such that $\hat{U} \vdash_{\rho} U_{0} \vdash_{\rho} U_{i}$ for all $i$.

Conversely, assume that $U$ satisfies

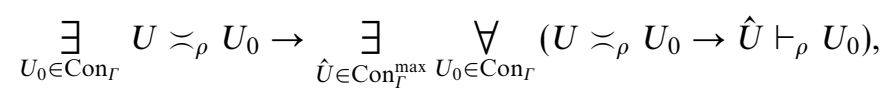

and let $U_{1}, U_{2} \in \operatorname{Con}_{\Gamma}$ be such that $U_{1} \asymp{ }_{\rho} U \asymp_{\rho} U_{2}$. From the assumption, we get a maximal $\hat{U} \in \operatorname{Con}_{\Gamma}^{\max }$ with $\hat{U} \vdash_{\rho} U_{i}$ for each $i$, so $U_{1} \asymp_{\rho} U_{2}$.

We will say the maximal extension of $U$ in $\Gamma$, if $U \in \mathrm{Con}_{\Gamma}^{\mathrm{ctr}}$, for the unique (up to equivalence) maximal neighbourhood entailing $U$; this we denote by $\hat{U}$, as in the statement of the above Lemma. But note that in the case of transitive neighbourhoods outside $\Gamma$ uniqueness is not guaranteed: an example with two maximals at type $\mathbb{D}$ is provided by the finite set $\Gamma=\{\mathrm{S} *, \mathrm{~S} 0, \mathrm{~S} 1\}$ and the neighbourhood $U=\{\mathrm{SS} *\}$. 


\subsection{Upper and middle application}

The notion of application $f x$ of some higher-type term $f$ to some input term $x$, both appropriately typed, is interpreted as 'the information that we hold on $x$ suffices to draw the information $f x$ on the output, given the information that we have on $f$.' In Section 2, in the definition of neighbourhood application, we saw that when we bring this notion down to the finite level it is entailment that we read into 'suffices,' but for our purposes, it will come in handy to consider a different version of application between neighbourhoods, where we replace entailment by consistency. As will become clear in the results below, starting already with Lemma 3.10, this kind of application is justified by the existence and use of transitive neighbourhoods.

Let $\Theta \in \operatorname{Fin}_{\rho \rightarrow \sigma}$ and $U \in \operatorname{Con}_{\rho}$. The (upper) application $\Theta \cdot U$ gathers all values $b \in$ Tok $_{\sigma}$ whose arguments $U_{b}$ fall under $U$ :

$$
b \in \Theta \cdot U:=\underset{U_{b} \in \operatorname{Con}_{\rho}}{\exists}\left(\left\langle U_{b}, b\right\rangle \in \Theta \wedge U \vdash_{\rho} U_{b}\right) .
$$

Note that this trivially generalises the neighbourhood application of Section 2; from now on, we will always write $W \cdot U$ instead of $W U$. The middle application $\Theta \cdot U$ is defined by

$$
b \in \Theta \cdot U:=\underset{U_{b} \in \operatorname{Con}_{\rho}}{\exists}\left(\left\langle U_{b}, b\right\rangle \in \Theta \wedge U \asymp_{\rho} U_{b}\right) .
$$

It follows immediately from the definition that the middle application yields at least as much information as the upper one does, namely $\Theta^{\cdot} U \subseteq \Theta \cdot U$.

In the case of a consistent left argument, we can make the following easy observations.

Lemma 3.7. Let $\rho, \sigma$ be arbitrary types, $W \in \mathrm{Con}_{\rho \rightarrow \sigma}, U \in \mathrm{Con}_{\rho}$ and $b \in \mathrm{Tok}_{\sigma}$.

1. We have $W \vdash_{\rho \rightarrow \sigma}\langle U, b\rangle$ if and only if $W \cdot U \vdash_{\sigma} b$.

2. We have $W \asymp_{\rho \rightarrow \sigma}\langle U, b\rangle$ if and only if $W \cdot U \asymp_{\sigma} b$.

Note that in Lemma 3.7 (2), the finite set $W \cdot U$ may not be consistent, but we still did not write $W \cdot U \asymp_{\sigma}^{F} b$; here, we are just saying that every pair $\left\{b^{W}, b\right\}$ will be consistent, for $b^{W} \in W \cdot U$.

Lemma 3.8 (Application). Let $\rho, \sigma$ be arbitrary types.

1. Application is consistently defined, that is, if $W \in \operatorname{Con}_{\rho \rightarrow \sigma}$ and $U \in \operatorname{Con}_{\rho}$, then $W \cdot U \in \mathrm{Con}_{\sigma}$.

2. Application is monotone in the right argument, in particular, if $\Theta \in \operatorname{Fin}_{\rho \rightarrow \sigma}$ and $U, U^{\prime} \in \operatorname{Con}_{\rho}$, with $U \vdash_{\rho} U^{\prime}$, then $\Theta \cdot U^{\prime} \subseteq \Theta^{\cdot} U$.

3. Application is monotone in the left argument, that is, if $\Theta, \Theta^{\prime} \in \operatorname{Fin}_{\rho \rightarrow \sigma}$ with $\Theta \vdash_{\rho \rightarrow \sigma}^{F}$ $\Theta^{\prime}$ and $U \in \operatorname{Con}_{\rho}$, then $\Theta \cdot U \vdash_{\sigma}^{F} \Theta^{\prime} \cdot U$.

Proof. For 1, let $W \in \operatorname{Con}_{\rho \rightarrow \sigma}$ and $U \in \operatorname{Con}_{\rho}$, and consider $b_{1}, b_{2} \in W \cdot U$. By the definition, there must exist $\left\langle U_{1}, b_{1}\right\rangle,\left\langle U_{2}, b_{2}\right\rangle \in W$, such that $U \vdash_{\rho} U_{1} \cup U_{2}$; it follows that $U_{1} \asymp{ }_{\rho} U_{2}$, so the consistency of $W$ ensures that $b_{1} \asymp{ }_{\sigma} b_{2}$.

For 2 , let $\Theta \in \operatorname{Fin}_{\rho \rightarrow \sigma}$ and $U, U^{\prime} \in \operatorname{Con}_{\rho}$, and assume that $U \vdash_{\rho} U^{\prime}$. Consider a $b \in V$; by the definition, there exists a $U_{b} \in L(\Theta)$ with $\left\langle U_{b}, b\right\rangle \in \Theta$ and $U^{\prime} \vdash_{\rho} U_{b}$; the assumption immediately gives $U \vdash_{\rho} U_{b}$, so $b \in \Theta^{\cdot} U$ as well. 
For 3, let $\Theta, \Theta^{\prime} \in \operatorname{Con}_{\rho \rightarrow \sigma}$ and $U \in \operatorname{Con}_{\rho}$, and assume that $\Theta \vdash_{\rho \rightarrow \sigma}^{F} \Theta^{\prime}$. Consider a $V^{\prime} \in \operatorname{Con}_{\Theta^{\prime}{ }^{\prime}}$; for each $b^{\prime} \in V^{\prime}$, there is a $U_{b^{\prime}} \in \operatorname{Con}_{\rho}$ such that $\left\langle U_{b^{\prime}}, b^{\prime}\right\rangle \in \Theta^{\prime}$ and $U \vdash_{\rho} U_{b^{\prime}}$; the set $W^{\prime}:=\left\{\left\langle U_{b^{\prime}}, b^{\prime}\right\rangle \in \Theta \mid b^{\prime} \in V^{\prime} \wedge U \vdash_{\rho} U_{b^{\prime}}\right\}$ is consistent in $\Theta^{\prime}$. By the assumption, there exists some $W \in \operatorname{Con}_{\Theta}$ such that $W \vdash_{\rho \rightarrow \sigma} W^{\prime}$. Since for each $\left\langle U_{b^{\prime}}, b^{\prime}\right\rangle \in W^{\prime}$, we have $W^{\cdot} U_{b^{\prime}} \vdash_{\sigma} b^{\prime}$, by 2 , we get $W \cdot U \vdash_{\sigma} W \cdot U_{b^{\prime}}$, hence, $W \cdot U \vdash_{\sigma} b^{\prime}$, that is, $W \cdot U \vdash_{\sigma} V^{\prime}$ and since $W \cdot U \in \mathrm{Con}_{\Theta} \cdot{ }^{\prime}$, we are done.

The following gives us conservative extensions of a neighbourhood by way of extending its set of arguments.

Lemma 3.9. Let $W \in \operatorname{Con}_{\rho \rightarrow \sigma}$ and $\Gamma \in \operatorname{Fin}_{\rho}$ such that $L(W) \subseteq \Gamma$. Then,

$$
W \sim_{\rho \rightarrow \sigma} \bigcup_{U \in \mathrm{Con}_{\Gamma}}\langle U, W \cdot U\rangle .
$$

Proof. From left to right, let $U \in \operatorname{Con}_{\Gamma}$ and $b \in W \cdot U$. There exists a $U_{b} \in \operatorname{Con}_{L(\Theta)}$ with $\left\langle U_{b}, b\right\rangle \in W$ and $U \vdash_{\rho} U_{b}$. Then, $\left\langle U_{b}, b\right\rangle \vdash_{\rho \rightarrow \sigma}\langle U, b\rangle$. The other way around is obvious, since $W \subseteq \bigcup_{U \in \operatorname{Con}_{\Gamma}}\langle U, W \cdot U\rangle$.

Turning our attention to middle application, the first thing we want to know is how it fares compared to Lemma 3.8 .

Lemma 3.10 (Middle application). Let $\rho, \sigma$ be arbitrary types.

1. Middle application is consistently defined for transitive right arguments, that is, if $W \in \operatorname{Con}_{\rho \rightarrow \sigma}$ and $U \in \operatorname{Con}_{\rho \mid L(W)}^{\mathrm{ctr}}$, then $W \cdot U \in \operatorname{Con}_{\sigma}$.

2. Middle application is antimonotone in the right argument, in particular, if $\Theta \in \operatorname{Fin}_{\rho \rightarrow \sigma}$ and $U, U^{\prime} \in \operatorname{Con}_{\rho}$ with $U \vdash_{\rho} U^{\prime}$, then $\Theta \cdot U \subseteq \Theta \cdot U^{\prime}$.

3. Middle application between neighbourhoods is monotone in the left argument for transitive right arguments, that is, if $W, W^{\prime} \in \operatorname{Con}_{\rho \rightarrow \sigma}$ are such that $W \vdash_{\rho \rightarrow \sigma} W^{\prime}$ and $U \in \operatorname{Con}_{\rho \mid L(W) \cup L\left(W^{\prime}\right)}^{\mathrm{ctr}}$, then $W \cdot U \vdash_{\sigma} W^{\prime} \cdot U$.

Proof. To show 1 , let $U \in \operatorname{Con}_{\rho \mid L(W)}^{\mathrm{ctr}}$ and $b_{1}, b_{2} \in W \cdot U$. Then, there exist $U_{1}, U_{2}$ with $\left\langle U_{i}, b_{i}\right\rangle \in W$ and $U_{i} \asymp_{\rho} U$. The transitivity of $U$ implies $U_{1} \asymp_{\rho} U_{2}$, and the consistency of $W$ ensures that $b_{1} \asymp_{\sigma} b_{2}$.

For 2, assume $U$ and $U^{\prime}$ such that $U \vdash_{\rho} U^{\prime}$, and let $b \in \Theta \cdot U$. There is some $U_{b}$ such that $\left\langle U_{b}, b\right\rangle \in \Theta$ and $U_{b} \asymp_{\rho} U$. By propagation (1), we get $U_{b} \asymp_{\rho} U^{\prime}$, so $b \in \Theta \cdot U^{\prime}$.

For 3. By 1, the assumption that $U \in \operatorname{Con}_{\rho \mid L(W) \cup L\left(W^{\prime}\right)}^{\mathrm{ctr}}$ ensures that the result of both middle applications is a neighbourhood (in general, if $\Gamma \subseteq \Gamma^{\prime}$, then $\operatorname{Con}_{\rho \mid \Gamma^{\prime}}^{\mathrm{ctr}} \subseteq \operatorname{Con}_{\rho \mid \Gamma}^{\mathrm{ctr}}$ ). Let $b^{\prime} \in W^{\prime} \cdot U$. By the definition of middle application, there exists a $\left\langle U^{\prime}, b^{\prime}\right\rangle \in W^{\prime}$, such that $U^{\prime} \asymp{ }_{\rho} U$. Since $W \vdash_{\rho \rightarrow \sigma} W^{\prime}$, there is a subneighbourhood $\left\{\left\langle U_{i}, b_{i}\right\rangle \mid i=1, \ldots, m\right\} \subseteq W$, such that for all $i=1, \ldots, m$, we have $U^{\prime} \vdash_{\rho} U_{i}$ and $\left\{b_{i} \mid i=1, \ldots, m\right\} \vdash_{\sigma} b^{\prime}$; by propagation, it follows that $U \asymp_{\rho} U_{i}$ for all $i$. This means that $b_{i} \in W \cdot U$ for all $i$, so $W \cdot U \vdash_{\sigma} b^{\prime}$, and we are done.

I The proof in fact shows that the equientailment here is linear ( $U$ entails $b$ linearly when $\{a\} \vdash b$ for some $a \in U$, see, Karádais (2018)). 
Lemma 3.11. Let $\Theta \in \operatorname{Fin}_{\rho \rightarrow \sigma}$.

1. For all $U, U^{\prime} \in \operatorname{Con}_{L(\Theta)}^{\mathrm{ctr}}$, if $U \asymp_{\rho} U^{\prime}$, then $\Theta \cdot U=\Theta \cdot U^{\prime}$.

2. For all $U \in \operatorname{Con}_{L(\Theta)}^{\mathrm{ctr}}$, we have $\Theta \cdot U=\Theta \cdot \hat{U}$.

3. For all $U \in \operatorname{Con}_{L(\Theta)}^{\max }$ and $U^{\prime} \in \operatorname{Con}_{\rho}$, if $U \asymp_{\rho} U^{\prime}$, then $\Theta \cdot U \subseteq \Theta \cdot U^{\prime}$.

Proof. For the first statement, assume that $U \asymp{ }_{\rho} U^{\prime}$ and let $b \in R(\Theta)$. We have $b \in \Theta \cdot U$ if and only if there is some $U_{b}$ with $\left\langle U_{b}, b\right\rangle \in \Theta$ and $U \asymp_{\rho} U_{b}$. Since $U$ is transitive in $L(\Theta)$, we get $U^{\prime} \asymp_{\rho} U_{b}$ by the assumption, so $b \in \Theta \cdot U^{\prime}$. The converse is similar.

For the second statement, let $U \in \operatorname{Con}_{L(\Theta)}^{\mathrm{ctr}}$. By the definition of middle application, if $b \in \Theta \cdot U$, then there is some $U_{b}$ with $\left\langle U_{b}, b\right\rangle \in \Theta$, such that $U_{b} \asymp{ }_{\rho} U$; since $U$ is transitive, by the maximality of its maximal extension, it follows that $\hat{U} \vdash_{\rho} U_{b}$, so the definition of application gives us $b \in \Theta \cdot \hat{U}$. For the other way around, if $b \in \Theta \cdot \hat{U}$, then there is a $U_{b}$ with $\left\langle U_{b}, b\right\rangle \in \Theta$, such that $\hat{U} \vdash_{\rho} U_{b}$; then $U \asymp{ }_{\rho} U_{b}$ by propagation (1), so $b \in \Theta \cdot U$, by the definition of middle application.

For the third statement, let $U$ be maximal in $L(\Theta)$ and $U^{\prime}$ some neighbourhood with $U \asymp_{\rho} U^{\prime}$. For every $b \in \Theta \cdot U$, by the definition of middle application, there is some $U_{b}$ with $\left\langle U_{b}, b\right\rangle \in \Theta$, such that $U \asymp_{\rho} U_{b}$, which by maximality means that $U \vdash_{\rho} U_{b}$; by propagation, we get $U^{\prime} \asymp_{\rho} U_{b}$, so $b \in \Theta \cdot U^{\prime}$, and we are done.

We close the section with a hint on how extremality evolves over types.

Lemma 3.12. Let $\Theta \in \operatorname{Fin}_{\rho \rightarrow \sigma}$ and $W \in \operatorname{Con}_{\Theta}$. We have $W \in \operatorname{Con}_{\Theta}^{\text {ctr }}$ if one of the following holds.

1. For all $U \in \operatorname{Con}_{L(\Theta)}^{\mathrm{ctr}}$, we have $W \cdot U \in \operatorname{Con}_{\Theta \cdot U}^{\mathrm{ctr}}$.

2. For all $U \in \operatorname{Con}_{L(\Theta)}^{\max }$, we have $W \cdot U \in \operatorname{Con}_{\Theta \cdot U}^{\mathrm{ctr}}$.

Proof. For the first criterion, let $\left\langle U_{i}, b_{i}\right\rangle \in \Theta$ be such that $\left\langle U_{i}, b_{i}\right\rangle \asymp_{\rho \rightarrow \sigma} W$ for $i=1,2$, and assume that $U_{1} \asymp_{\rho} U_{2}$. Consider a $U \in \operatorname{Con}_{L(\Theta)}^{\text {ctr }}$ with $U \asymp_{\rho} U_{1} \cup U_{2}$; then $b_{i} \in \Theta \cdot U$ for each $i$. From $\left\langle U_{i}, b_{i}\right\rangle \asymp_{\rho \rightarrow \sigma} W$, by Lemma 3.10 (1), we get $b_{i} \asymp_{\sigma} W \cdot U$ for both $i$, so by the assumption, we get $b_{1} \asymp{ }_{\sigma} b_{2}$.

To get the second criterion, it suffices to show that

$$
\underset{U \in \operatorname{Con}_{L(\Theta)}^{\max }}{\forall} W \cdot U \in \operatorname{Con}_{\Theta \cdot U}^{\mathrm{ctr}} \rightarrow \underset{U \in \operatorname{Con}_{L(\theta)}^{\mathrm{ctr}}}{\forall} W \cdot U \in \operatorname{Con}_{\Theta \cdot U}^{\mathrm{ctr}} \cdot
$$

Assume that $W$ is such that $W \cdot U \in \operatorname{Con}_{\Theta \cdot U}^{\mathrm{ctr}}$, for all $U \in \operatorname{Con}_{L(\Theta)}^{\max }$, and let $U \in \operatorname{Con}_{L(\Theta)}^{\mathrm{ctr}}$. Consider the maximal extension $\hat{U}$ of $U$. On the one hand, we have $\hat{U} \in \operatorname{Con}_{L(\Theta)}^{\max }$, so $W \cdot \hat{U} \in \operatorname{Con}_{\Theta \cdot \hat{U}}^{\mathrm{ctr}}$ by the assumption. On the other hand, we have $\hat{U} \in \operatorname{Con}_{L(\Theta)}^{\mathrm{ctr}}$ with $\hat{U} \asymp \rho U$, so $\Theta \cdot \hat{U}=\Theta \cdot U$ and $W \cdot \hat{U}=W \cdot U$, by Lemma 3.11 (1). It follows that $W \cdot U \in \operatorname{Con}_{\Theta \cdot U}^{\mathrm{ctr}}$, so we can apply the previous criterion and we are done.

\section{Totality of neighbourhoods}

In this section, we take the first two steps of the strategy that we outlined in Section 1. A total object of type $\rho \rightarrow \sigma$ is represented by a possibly infinite token set that (a) is an ideal, that is, consistent and deductively closed, (b) admits all totals of type $\rho$ as arguments - a 
property we think of as 'omniception,' for lack of a less pompous synonym for admission (or acceptance) of all, that would be as grammatically smooth - and (c) responds to every total argument with a total value at type $\sigma$. To bring the notion down to the finite level, we dispose of half of the demand (a), namely, that the set of tokens be deductively closed, and we reinterpret 'admittance' and 'response' in (b) and (c) in terms of consistency rather than entailment. The first move, which is clearly dictated by the demand of finiteness, in some sense causes the reaction of the second move: what we lose by denying deductive closure, we have to regain with the wider and more tolerant scope of consistency.

\subsection{Finite density}

At type $\rho$, a side extension of a neighbourhood $U$ will be any neighbourhood $U^{\prime}$ that is consistent with $U$. We give a name to this rather mundane notion just to point to its intended use: trivially, if $U^{\prime}$ is a side extension of $U$, then $U^{\prime} \cup U$ is an extension of $U$, and this is exactly how we will work towards finding total extensions of neighbourhoods.

Lemma 4.1. Let $\rho$ and $\sigma$ be types. For every $W \in \operatorname{Con}_{\rho \rightarrow \sigma}$ and for every mapping $V \mapsto V^{\prime}$ of neighbourhoods $V \in \mathrm{Con}_{\sigma}$ to side extensions thereof, the finite set $\bigcup_{U \in \operatorname{Con}_{L(W)}^{\mathrm{ctr}}}\left\langle U,(W \cdot U)^{\prime}\right\rangle$ is a side extension of $W$.

Proof. To show the consistency of the finite set, let $\left\langle U_{i}, b_{i}\right\rangle$ be such that $U_{i} \in \operatorname{Con}_{L(W)}^{\mathrm{ctr}}$ and $b_{i} \in\left(W \cdot U_{i}\right)^{\prime}$, for $i=1,2$. If $U_{1} \asymp{ }_{\rho} U_{2}$, then $W \cdot U_{1}=W \cdot U_{2}$ by Lemma 3.11 (1), hence, $\left(W \cdot U_{1}\right)^{\prime}=\left(W \cdot U_{2}\right)^{\prime}$, and $b_{1} \asymp{ }_{\sigma} b_{2}$.

To show the side extension, let $\langle U, b\rangle \in W$ and $\left\langle U^{\prime}, b^{\prime}\right\rangle$ be such that $U^{\prime} \in \operatorname{Con}_{L(W)}^{\mathrm{ctr}}$ and $b^{\prime} \in(W \cdot U)^{\prime}$. If $U \asymp_{\rho} U^{\prime}$, then by the definition of middle application, we have $b \in W \cdot U$. But $W \cdot U \asymp_{\sigma}(W \cdot U)^{\prime}$ by assumption, so $b \asymp_{\sigma} b^{\prime}$.

As we mentioned in Section 1, a total token at a base type $\imath$ is a token $p \in$ Tok $_{l}$ which consists exclusively of proper constructors; write $p \in \operatorname{Tok}_{l}^{g}$. We have $C p_{1} \cdots p_{r} \in \operatorname{Tok}_{l}^{g}$ if and only if $C$ is a proper constructor of arity $r$ and $p_{i} \in \operatorname{Tok}_{l}^{g}$ for all $i=1, \ldots, r$. So, $\mathrm{SB} * 0 \notin \mathrm{Tok}_{\mathbb{D}}^{g}$ but SB10 $\in \mathrm{Tok}_{\mathbb{D}}^{g}$. Define total neighbourhoods inductively over types

$$
\begin{aligned}
U \in \operatorname{Con}_{l}^{g} & :=\underset{p \in \operatorname{Tok}_{l}^{g}}{\exists} U \vdash_{l} p, \\
W \in \operatorname{Con}_{\rho \rightarrow \sigma}^{g}: & =\underset{P \in \operatorname{Con}_{\rho}^{g}}{\forall} W \cdot P \in \operatorname{Con}_{\sigma}^{g} .
\end{aligned}
$$

Examples of total neighbourhoods are $\{\mathrm{B} 0 *, \mathrm{~B} * 1\}$ and $\{\mathrm{B} 0 \mathrm{~S} 0\}$ at type $\mathbb{D}$, and the neighbourhood $\{\langle\{0\}\rangle, 0,\langle\{\mathrm{~S} *\}, \mathrm{S} 0\rangle\}$ at type $\mathbb{N} \rightarrow \mathbb{N}$, which we saw in Section 1 . A type 2 example would be the neighbourhood

$$
X=\{\langle\{\langle\{t t\}, 0\rangle\}, 0\rangle,\langle\{\langle\{t t\}, \mathrm{S} *\rangle\}, \mathrm{S} 0\rangle\}
$$

at type $(\mathbb{B} \rightarrow \mathbb{N}) \rightarrow \mathbb{N}$ : Let $T$ be a total neighbourhood at type $\mathbb{B} \rightarrow \mathbb{N}$; for the total singleton $\{t t\}$ of type $\mathbb{B}$, we get $T \cdot\{t t\} \vdash_{\mathbb{N}} S^{m} 0$ for some $m \geqslant 0$, so we either have $T \cdot\{t t\} \asymp_{\mathbb{N}} 0$ or $T \cdot\{t t\} \asymp_{\mathbb{N}} \mathrm{S}^{*}$, which by Lemma 3.7 (2) means either $T \asymp_{\mathbb{B} \rightarrow \mathbb{N}}\langle\{t\}, 0\rangle$ or $T \asymp \mathbb{B} \rightarrow \mathbb{N}\langle\{t t\}, S *\rangle$; in the first case, we get $X \cdot T=\{0\}$ and in the second case, $X \cdot T=\{\mathrm{S} 0\}$, both of which are total neighbourhoods at type $\mathbb{N}$, as we wanted. 
The arguments of a higher-type total neighbourhood form a finite set that can accept (in the sense of middle application) every total neighbourhood of the source type, in a way that ensures their safe (that is, consistency-respecting) allotment to an appropriate value. Define weakly omniceptive finite sets explicitly by

$$
\Gamma \in \operatorname{Fin}_{\rho}^{w o}:=\underset{P \in \operatorname{Con}_{\rho}^{\mathrm{g}} U^{P} \in \operatorname{Con}_{\Gamma}^{\mathrm{ctr}}}{\exists} \asymp_{\rho} U^{P} .
$$

At a type $\rho \rightarrow \sigma$, call $\Theta$ a (strongly) omniceptive finite set, and write $\Theta \in \operatorname{Fin}_{\rho \rightarrow \sigma}^{o}$, if

$$
\Theta \in \operatorname{Fin}_{\rho \rightarrow \sigma}^{w o} \wedge \underset{U \in \operatorname{Con}_{L(\Theta)}^{\max }}{\forall}\left(U \in \operatorname{Con}_{\rho}^{g} \wedge \Theta \cdot U \in \operatorname{Fin}_{\sigma}^{o} \wedge\langle U, \Theta \cdot U\rangle \subseteq \Theta\right),
$$

which intuitively says that, beyond weak omniception, $\Theta$ must meet certain requirements of finite totality, preservation of omniception and closure under middle application for each of its left maximals. By convention, we set $\operatorname{Fin}_{l}^{o}:=$ Fin $_{l}^{w o}$ for arbitrary base types. In the following, we always use the term 'omniceptive' (without a qualifier) to mean 'strongly omniceptive' (which for base types is the same as 'weakly omniceptive'). A further convention that we will often employ in subsequent arguments is to use lower case 'g,' 'wo,' and 'o' as superscripts of terms: for example, we may write ' $U^{g}$ ' for a neighbourhood that is to belong to $\mathrm{Con}^{g}$ and possibly relates to a previously discussed $U$, or ' $\Gamma^{o}$ ' for a finite set that is to belong to Fin $^{o}$ and possibly relates to a previously introduced $\Gamma$.

Call a type $\rho$ finitely dense if every neighbourhood $U$ at $\rho$ has a total side extension $U^{g}$, and finitely omniceptive if every finite set $\Gamma$ has an omniceptive extension $\Gamma^{o}$. Moreover, call it finitely total-transitive if every total neighbourhood $U$ is transitive (in $\rho$ ). The latter just means that $U_{1} \asymp{ }_{\rho} U \asymp_{\rho} U_{2}$ implies $U_{1} \asymp{ }_{\rho} U_{2}$ for all $U_{1}, U_{2} \in \mathrm{Con}_{\rho}$, and we write $U \in \operatorname{Con}_{\rho}^{\mathrm{ctr}}$. Here is a lemma to set the intuition straight.

Lemma 4.2 (Compactness of transitivity). Let $\rho$ be a type. A neighbourhood is transitive in $\rho$ if and only if it is transitive for every finite set of $\rho$.

Proof. For the less trivial direction, let $U \in \operatorname{Con}_{\rho}$ be such that $U \in \operatorname{Con}_{\rho \mid \Gamma}^{\mathrm{ctr}}$ for every $\Gamma \in \operatorname{Fin}_{\rho}$, and let $U_{1}, U_{2} \in \operatorname{Con}_{\rho}$ be such that $U_{1} \asymp{ }_{\rho} U \asymp_{\rho} U_{2}$. Set $\Gamma:=U_{1} \cup U \cup U_{2}$; then, we have $U_{1} \asymp{ }_{\rho} U_{2}$ by $\Gamma$-transitivity.

To start off the main argument, we need some elementary definitions and observations concerning base types. The size $\|a\|$ of a base-type token $a \in \operatorname{Tok}_{l}$ counts the proper constructors of the token: $\|*\|=0$ and $\left\|C a_{1} \cdots a_{r}\right\|=1+\left\|a_{1}\right\|+\cdots+\left\|a_{r}\right\|$. It is an easy induction to show that $\{a\} \vdash_{l} b$ implies $\|a\| \geqslant\|b\|$ for all tokens $a, b$. The supremum or eigentoken $\sup (U)$ of a base-type neighbourhood $U \in \operatorname{Con}_{l}$ is defined by $\sup \left(\varnothing_{l}\right)=*_{l}, \sup (\{a\})=a$ and $\sup \left(\left\{a_{1}, \ldots, a_{m}\right\}\right)=\sup ^{t}\left(\cdots \sup ^{t}\left(a_{1}, a_{2}\right) \cdots, a_{m}\right)$ for $m>1$, where $\sup ^{t}(a, *)=a$ and $\sup ^{t}\left(C a_{1} \cdots a_{r}, C b_{1} \cdots b_{r}\right)=C \sup ^{t}\left(a_{1}, b_{1}\right) \cdots \sup ^{t}\left(a_{r}, b_{r}\right)($ we do not need to define the auxiliary mapping sup ${ }^{t}$ on inconsistent pairs). Again, an easy induction shows that $U \sim_{l}\{\sup (U)\}$ for all $U \in \operatorname{Con}_{l}$; in particular, we can represent every total neighbourhood $P \in \operatorname{Con}_{l}^{g}$ by its total eigentoken $\sup (P) \in \operatorname{Tok}_{l}^{g}$.

Proposition 4.3. Every base type is finitely total-transitive, finitely dense and finitely omniceptive. 
Proof. Let $l$ be any base type with a distinguished nullary constructor 0 . For the transitivity of total neighbourhoods, let $P \in \operatorname{Con}_{l}^{g}$ and $U_{1}, U_{2} \in \operatorname{Con}_{l}$ be such that $U_{i} \asymp_{l} P$ for each $i$. Then, $P \vdash_{l} U_{i}$, for both $i=1,2$, since, as is easy to see, total tokens are maximal at base types, so $U_{1} \asymp{ }_{1} U_{2}$.

We turn to finite density by first considering tokens: the trivial token $*$ is consistent with 0 , and if $p_{1}, \ldots, p_{r}$ are total tokens consistent with $a_{1}, \ldots, a_{r}$, respectively, then $C p_{1} \cdots p_{r}$ is a total token consistent with $C a_{1} \cdots a_{r}$, for an $r$-ary constructor $C$. Finally, if $U \in \operatorname{Con}_{l}$, and $p$ is a total token consistent to $\sup (U)$, then the neighbourhood $\overline{\{p\}}$ is obviously a total neighbourhood consistent with (above, even) $U$.

Before we turn to the finite omniception, we need some auxiliary facts. First, we claim that

$$
\underset{a \in \operatorname{Tok}_{l}}{\forall} \underset{p \in \operatorname{Tok}_{l}^{g}}{\forall}\left(\|a\|>\|p\| \rightarrow a \Varangle_{l} p\right) .
$$

Indeed, let $p$ be a total token and $a$ an arbitrary token. In case, $p$ consists of a single nullary constructor, then $\|p\|=1$ and there have to exist a constructor $C$ with arity $r>0$ and further tokens $a_{1}, \ldots, a_{r}$ such that $a=C a_{1} \cdots a_{r}$ (with $\left\|a_{i}\right\|>0$ for at least some $i$ ); we have $a \Varangle_{l} p$ by the definition of consistency. In case, $p=C p_{1} \cdots p_{r}$ for some total tokens $p_{1}, \ldots, p_{r}$, then $a$ will either start with a different (nonnullary) head constructor than $C$, in which case we are done, or there must exist $a_{1}, \ldots, a_{r}$ such that $a=C a_{1} \cdots a_{r}$; from $\|a\|>$ $\|p\|$ it follows that $\sum_{i}\left\|a_{i}\right\|>\sum_{i}\left\|p_{i}\right\|$, so there must exist at least some $i$ with $\left\|a_{i}\right\|>\left\|p_{i}\right\|$; by the induction hypothesis, we know that this means that $a_{i} \Varangle_{1} p_{i}$, therefore, $a \Varangle_{1} p$.

Now, for a fixed total token $p \in \operatorname{Tok}_{l}^{g}$ and a fixed natural number $n$, let $U^{p, n}$ stand for the finite set $\left\{a \in \operatorname{Tok}_{l} \mid a \asymp_{l} p \wedge\|a\| \leqslant n\right\}$; this is a neighbourhood thanks to the transitivity of the total $p$. Note that for every $p$, we have $U^{p, 0}=\left\{*_{l}\right\}$, and also, due to (3), for all $n \geqslant\|p\|$, we have $U^{p, n}=U^{p,\|p\|}$. Now, either $p$ consists of a single nullary constructor or not; in case it does, then $U^{p, 1}=\left\{{ }_{l}, p\right\}$, while if $p=C p_{1} \cdots p_{r}$ for a constructor $C$ with arity $r>0$ and total tokens $p_{1}, \ldots, p_{r}$, then, we claim that the component neighbourhoods satisfy

$$
\underset{i=1}{\forall} U^{p, n}(i)=U^{p_{i}, n-1} .
$$

Indeed, fix an $i$. From left to right, assume that $a^{i} \in U^{p, n}(i)$; this means that there exists an $a \in U^{p, n}$ such that $a(i)=a^{i}$; by the definition of $U^{p, n}$, we know that $a \asymp_{l} p$, which implies that $a^{i} \asymp_{l} p_{i}$; we also know that $\|a\| \leqslant n$, so $\left\|a^{i}\right\| \leqslant\|a\|-1 \leqslant n-1$, therefore, $a^{i} \in U^{p_{i}, n-1}$. For the other way around, assume that $a^{i} \in U^{p_{i}, n-1}$, that is, that $a^{i} \asymp_{l} p_{i}$ and $\left\|a^{i}\right\| \leqslant n-1$; consider the token $a:=C \vec{*} a^{i} \vec{*}$ (which has $a^{i}$ as its $i$ th component token and stars everywhere else); it obviously satisfies $a \asymp_{l} p$, and since $\left\|a^{i}\right\| \leqslant n-1$, it also satisfies $\|a\| \leqslant n$, therefore, $a \in U^{p, n}$; it follows that $a(i)=a^{i} \in U^{p, n}(i)$.

Finally, we claim that the following holds for a fixed $n$ :

$$
\underset{a \in \operatorname{Tok}_{l}}{\forall} \underset{p \in \operatorname{Tok}_{l}^{g}}{\forall}\left(\|a\|=n \leqslant\|p\| \wedge a \asymp{ }_{l} U^{p, n} \rightarrow U^{p, n} \vdash_{l} a\right) .
$$

Indeed, let $p$ be some total token and $a$ an arbitrary one, such that $\|a\|=n \leqslant\|p\|$ and $a \asymp_{l} U^{p, n}$. In case $n=0$, we necessarily have $a=*_{l} \in U^{p, 0}$. In case $n>0$, then for some constructor $C$ of the algebra $l$ with arity $r$ there exist total tokens $p_{1}, \ldots, p_{r}$ such that $p=C p_{1} \cdots p_{r}$, and since $C^{*} \in U^{p, n}$ and $a \asymp_{l} U^{p, n}$, there exist tokens $a_{1}, \ldots, a_{r}$ such that 
$a=C a_{1} \cdots a_{r}$; for every $i=1, \ldots, r$, since $a \asymp_{l} U^{p, n}$, we have $a_{i} \asymp_{l} U^{p, n}(i)$, which by (4) means that $a_{i} \asymp_{l} U^{p_{i}, n-1}(i)$, while from the assumption that $\|a\|=n$, we get $\left\|a_{i}\right\| \leqslant n-1$; the induction hypothesis yields $U^{p_{i}, n-1} \vdash_{l} a_{i}$ for every $i$; so, we get

$$
U^{p, n} \sim_{l} C U^{p, n}(1) \cdots U^{p, n}(r) \stackrel{(4)}{=} C U^{p_{1}, n-1} \cdots U^{p_{r}, n-1} \vdash_{l} C a_{1} \cdots a_{r}=a,
$$

and we are done.

Now for the finite omniception. If $\Gamma$ is trivial (that is, if it carries no proper information), then set $\Gamma^{o}:=\{*\}$. If not, let $m:=\max \left\{\|\sup (U)\| \mid U \in \operatorname{Con}_{\Gamma}\right\}$, and set $\Gamma^{o}:=\left\{a \in \operatorname{Tok}_{l} \mid\|a\| \leqslant m\right\}$. Consider a total neighbourhood $P$ and let $p:=\sup (P)$. In case $\|p\| \leqslant m$, we have $p \in \Gamma^{o}$, so it suffices to set $U^{P}:=\{p\}$. In case $\|p\|>m$, we set $U^{P}:=U^{p, m}$; by the construction of $\Gamma^{o}$, we have $U^{p, m} \in \operatorname{Con}_{\Gamma^{o}}$, and we also have that $U^{p, m}$ is transitive in $\Gamma^{o}$ : for any two tokens $b_{1}, b_{2} \in \Gamma^{o}$ with $b_{1} \asymp_{l} U^{p, m} \asymp_{l} b_{2}$, since $\left\|b_{i}\right\| \leqslant m<\|p\|$ for both $i$, we get $\in U^{p, m} \vdash_{l} b_{i}$ from (5), so $b_{1} \asymp{ }_{l} b_{2}$.

Proposition 4.4 (Finite total-transitivity). Let $\rho$ and $\sigma$ be finitely total-transitive types. If $\rho$ is finitely dense, then $\rho \rightarrow \sigma$ is finitely total-transitive.

Proof. Let $T \in \operatorname{Con}_{\rho \rightarrow \sigma}^{g}$ and $W_{1}, W_{2} \in \operatorname{Con}_{\rho \rightarrow \sigma}$, with $W_{1} \asymp_{\rho \rightarrow \sigma} T \asymp_{\rho \rightarrow \sigma} W_{2}$. Consider pairs $\left\langle U_{i}, b_{i}\right\rangle \in W_{i}, i=1,2$, and assume that $U_{1} \asymp{ }_{\rho} U_{2}$. By the finite density at $\rho$, there exists a $P \in \operatorname{Con}_{\rho}^{g}$, such that $P \asymp{ }_{\rho} U_{1} \cup U_{2}$. By the assumptions at $\rho$ and Lemma 3.10 (1), we get $b_{1} \asymp_{\sigma} T \cdot P \asymp_{\sigma} b_{2}$. But $T \cdot P$ is total, so the assumption at $\sigma$ gives $b_{1} \asymp_{\sigma} b_{2}$.

Proposition 4.5 (Finite density). Let $\rho$ and $\sigma$ be types. If $\rho$ is finitely omniceptive and $\sigma$ finitely dense and finitely total-transitive, then $\rho \rightarrow \sigma$ is finitely dense.

Proof. Let $W \in \operatorname{Con}_{\rho \rightarrow \sigma}$ be any neighbourhood. By finite omniception at $\rho$, we get a $\Gamma \in \operatorname{Fin}_{\rho}^{o}$ with $L(W) \subseteq \Gamma$. Consider the neighbourhood $W^{o}:=\bigcup_{U \in \operatorname{Con}_{\Gamma}}\langle U, W \cdot U\rangle$; by Lemma 3.9, we have $W \sim_{\rho \rightarrow \sigma} W^{o}$. Now set

$$
W^{g}:=\bigcup_{U \in \operatorname{Con}_{L\left(W^{o}\right)}^{\mathrm{ctrr}}}\left\langle U,\left(W^{o} \cdot U\right)^{g}\right\rangle,
$$

with the help of density at $\sigma$; note that $L\left(W^{g}\right)=L\left(W^{o}\right)=\Gamma$. This is a side extension of $W^{o}$ (therefore of $W$ as well) by Lemma 4.1 .

To show that it is total, let $P \in \operatorname{Con}_{\rho}^{g}$. Since $L\left(W^{g}\right)$ is omniceptive (in fact, that it is weakly omniceptive is enough), there is some $U^{P} \in \operatorname{Con}_{\Gamma}^{\text {ctr }}$ such that $P \asymp{ }_{\rho} U^{P}$. We have $\left\langle U^{P},\left(W^{o} \cdot U^{P}\right)^{g}\right\rangle \subseteq W^{g}$ by construction, and $W^{g} \cdot P=\left(W^{o} \cdot U^{P}\right)^{g}$, since, by transitivity of total neighbourhoods at $\sigma$, the value $W^{o} \cdot P$ is independent from the choice of $U^{P}$.

Proposition 4.6 (Finite omniception). Let $\rho$ and $\sigma$ be finitely total-transitive types. If $\rho$ is finitely dense and $\sigma$ finitely omniceptive, then $\rho \rightarrow \sigma$ is finitely omniceptive.

Proof. Let $\Theta \in \operatorname{Fin}_{\rho \rightarrow \sigma}$ be any finite set. Extend it as follows:

$$
\Theta^{o}:=\Theta \cup \bigcup_{U \in \operatorname{Con}_{L(\Theta)}^{\max }}\left\langle U^{g},(\Theta \cdot U)^{o}\right\rangle,
$$

with the use of finite density at $\rho$ and finite omniception at $\sigma$. 
If we show that this is weakly omniceptive, then it will be omniceptive immediately by construction (based on Lemma 3.5). Let $T \in \operatorname{Con}_{\rho \rightarrow \sigma}^{g}$. For every $U \in \operatorname{Con}_{L(\Theta)}^{\max }$, we have $T \cdot U^{g} \in \operatorname{Con}_{\sigma}^{g}$, and since $(\Theta \cdot U)^{o}$ is omniceptive, there will be some $V^{T \cdot U^{g}} \in \operatorname{Con}_{(\Theta \cdot U)^{o}}^{\text {ctr }}$, such that $T \cdot U^{g} \asymp_{\sigma} V^{T \cdot U^{g}}(\star)$. Fix these side extensions and set

$$
W^{T}:=\bigcup_{U \in \operatorname{Con}_{L\left(\Theta^{o}\right)}^{\max }}\left\langle U^{g}, V^{T \cdot U^{g}}\right\rangle .
$$

We have $W^{T} \subseteq \Theta^{o}$ by construction. Moreover, we have $T \asymp{ }_{\rho \rightarrow \sigma} W^{T}$ : let $\langle U, b\rangle \in T$ and $\left\langle U^{\prime}, b^{\prime}\right\rangle \in W^{T}$ be such that $U \asymp{ }_{\rho} U^{\prime}$; we have $b \in T \cdot U^{\prime}$ and $b^{\prime} \in V^{T \cdot U^{\prime}}$, so $b \asymp_{\sigma} b^{\prime}$ by $(\star)$. Since $T$ is total, $W^{T}$ is a neighbourhood by transitivity of total neighbourhoods, which we get for $\rho \rightarrow \sigma$ by Proposition 4.4. Finally, it is transitive in $\Theta^{o}$ by Lemma 3.12 (2), since for every $U \in \operatorname{Con}_{L(\Theta)}^{\max }$, we have by construction $W^{T} \cdot U=V^{T \cdot U}$, which is transitive in $\Theta \cdot U$ by omniception.

Theorem 4.7. Every type is finitely omniceptive, finitely total-transitive, and, in particular, finitely dense.

Proof. We get this by mutual induction over types from Propositions 4.3-4.6.

\subsection{Totality of transitive neighbourhoods}

There is plenty of evidence to suggest that total neighbourhoods at $\rho$ are to $\operatorname{Con}_{\rho}$ what transitive neighbourhoods in $\Gamma$ are to $\operatorname{Con}_{\Gamma}$. For one, Theorem 4.7 shows that total neighbourhoods are transitive. Furthermore, an immediate corollary of total transitivity is that consistency, restricted to the total neighbourhoods, becomes an equivalence relation, that is,

$$
\underset{P_{1}, P_{2}, P_{3} \in \mathrm{Con}_{\rho}^{g}}{\forall}\left(P_{1} \asymp_{\rho} P_{2} \asymp_{\rho} P_{3} \rightarrow P_{1} \asymp_{\rho} P_{3}\right) .
$$

Here, are further examples of using total transitivity, which include some more evidence to this effect.

Lemma 4.8. Let $\rho$ and $\sigma$ be types. Let $\Theta \in \operatorname{Fin}_{\rho \rightarrow \sigma}, P, P^{\prime} \in \operatorname{Con}_{\rho}^{g}$, and $U, U^{\prime} \in \operatorname{Con}_{\rho}$.

1. For every $U^{P} \in \operatorname{Con}_{L(\Theta)}$ with $P \asymp{ }_{\rho} U^{P}$, we have $\Theta \cdot P \subseteq \Theta \cdot U^{P}$. Moreover, we have $\Theta \cdot P=\Theta \cdot U^{P}$ whenever $U^{P} \in \operatorname{Con}_{L(\Theta)}^{\max }$.

2. If $P \asymp{ }_{\rho} P^{\prime}$, then $\Theta \cdot P=\Theta \cdot P^{\prime}$.

3. If $U \in \operatorname{Con}_{\rho}^{g}$ and $U^{\prime} \vdash_{\rho} U$, then $U^{\prime} \in \operatorname{Con}_{\rho}^{g}$.

4. If $T \in \operatorname{Con}_{\rho \rightarrow \sigma}^{g}$ and $U \in \operatorname{Con}_{L(T)}^{\mathrm{ctr}}$, then $T \cdot U \in \operatorname{Con}_{\sigma}^{g}$.

Proof. For 1 , let $b \in \Theta \cdot P$. Then, by the definition of middle application, there is some $U$ with $\langle U, b\rangle \in \Theta$, such that $U \asymp{ }_{\rho} P$. From $U \asymp{ }_{\rho} P \asymp{ }_{\rho} U^{P}$, we get $U \asymp_{\rho} U^{P}$ by Theorem 4.7, so the definition of middle application yields that $b \in \Theta \cdot U^{P}$. Moreover, if $U^{P}$ is actually maximal in $L(\Theta)$, then, by Lemma 3.11 (3), we immediately get $\Theta \cdot U^{P} \subseteq \Theta \cdot P$.

For 2, assume that $P \asymp{ }_{\rho} P^{\prime}$ and let $b \in \Theta \cdot P$. By the definition of middle application, there is some $U$ with $\langle U, b\rangle \in \Theta$, such that $U \asymp \rho P$. By Theorem 4.7 and the assumption, we have $U \asymp{ }_{\rho} P^{\prime}$, so $b \in \Theta \cdot P^{\prime}$. 
For 3. At a base type $\imath$ if $U \in \mathrm{Con}_{l}^{g}$, then there exists a total token $p$ such that $U \vdash_{l} p$. The transitivity of entailment yields what we need. At a higher type $\rho \rightarrow \sigma$, let $W \in \operatorname{Con}_{\rho \rightarrow \sigma}^{g}$ and $W^{\prime} \vdash_{\rho \rightarrow \sigma} W$. Let further $P \in \operatorname{Con}_{\rho}^{g}$. By Theorem 4.7, $P$ is transitive for $L(W)$, so by the left monotonicity of middle application on transitive arguments (Lemma 3.10 (3)), we have $W^{\prime} \cdot P \vdash_{\sigma} W \cdot P$, and by the totality of $W$, we get $W \cdot P \in \operatorname{Con}_{\sigma}^{g}$, so the induction hypothesis at $\sigma$ finishes the job.

For 4. By Theorem 4.7 there exists some $P^{U} \in \operatorname{Con}_{\rho}^{g}$ with $P^{U} \asymp_{\rho} U$. By 1 , we have $T \cdot P^{U} \subseteq T \cdot U$, where $T \cdot U$ is consistent by Lemma 3.10 (1). It follows by 3 that $T \cdot U \in \operatorname{Con}_{\sigma}^{g}$.

Note in particular that Lemma 4.8 (3) is analogous to Lemma 3.3 (both of them actually anticipate Lemma 5.2).

We now show that the correspondence between transitivity and finite totality is complete.

Theorem 4.9 (Explicit finite totality). At every type, a neighbourhood is total if and only if it is transitive.

Proof by induction over types. The rightward direction we have of course from Theorem 4.7. For the other direction, we have to show that, at each type, every transitive neighbourhood must be total.

At a base type $l$, assume that $U \in \operatorname{Con}_{l}^{\text {ctr }}$. Obviously, we have $U \chi_{l}\{*\}$, so there will be a constructor $C$ and tokens $a_{1}, \ldots, a_{r} \in \operatorname{Tok}_{l}$ such that $U \sim_{l}\left\{C a_{1} \cdots a_{r}\right\}$ (its supremum). By Lemma 3.4 (2), since $U$ is transitive, for any two tokens $b_{1}, b_{2} \in \mathrm{Tok}_{l}$, we will have $b_{1} \asymp_{l} U \asymp_{l} b_{2}$ imply $b_{1} \asymp_{l} b_{2}$. Then, for $i=1, \ldots, r$, we have

$$
\begin{aligned}
b_{1 i} \asymp_{l} a_{i} \asymp_{l} b_{2 i} & \Rightarrow C a_{1} \cdots b_{1 i} \cdots a_{r} \asymp_{l} U \asymp_{l} C a_{1} \cdots b_{2 i} \cdots a_{r} \\
& \stackrel{\text { ctr }}{\Rightarrow} C a_{1} \cdots b_{1 i} \cdots a_{r} \asymp_{l} C a_{1} \cdots b_{2 i} \cdots a_{r} \\
& \Rightarrow b_{1 i} \asymp_{l} b_{2 i},
\end{aligned}
$$

for any $b_{1 i}, b_{2_{i}} \in \operatorname{Tok}_{l}$, which by induction hypothesis yields $a_{i} \in \operatorname{Tok}_{l}^{g}$. It follows that $C a_{1} \cdots a_{r}$ itself is a total token, so $U$ is a total neighbourhood.

At type $\rho \rightarrow \sigma$, assume that $W \in \operatorname{Con}_{\rho \rightarrow \sigma}^{\mathrm{ctr}}$, and let $P \in \mathrm{Con}_{\rho}^{g}$. For any $b_{1}, b_{2} \in \mathrm{Tok}_{\sigma}$, we have

$$
\begin{aligned}
b_{1} \asymp_{\sigma} W \cdot P \asymp_{\sigma} b_{2} & \Leftrightarrow\left\langle P, b_{1}\right\rangle \asymp_{\rho \rightarrow \sigma} W \asymp_{\rho \rightarrow \sigma}\left\langle P, b_{2}\right\rangle \\
& \stackrel{\text { ctr }}{\Rightarrow}\left\langle P, b_{1}\right\rangle \asymp_{\rho \rightarrow \sigma}\left\langle P, b_{2}\right\rangle \\
& \Rightarrow b_{1} \asymp_{\sigma} b_{2},
\end{aligned}
$$

which means that $W \cdot P$ is transitive in $\sigma$, so by the induction hypothesis at $\sigma$, we get $W \cdot P \in \mathrm{Con}_{\sigma}^{g}$, and by the definition of finite totality, we have $W \in \mathrm{Con}_{\rho \rightarrow \sigma}^{g}$, as we wanted.

The theorem indicates that our notion of finite totality is a robust one. In the next section, we will see how we can connect it to the traditional notion of totality for ideals. Interestingly, we will see that its equivalence to transitivity is peculiar to the finitary level: in Proposition 5.12, the respective correspondence for ideals is shown to be tilted. 


\section{Elevating totality to ideals}

The last step in our strategy is to find a canonical extension of a total neighbourhood to a total ideal. The natural candidate would be the deductive closure of a neighbourhood, but again, closure under entailment is too strict for our purposes, since it presents recurring technical difficulties stemming from the fact that the application of ideals is an 'upper' one, while we have defined finite totality in terms of middle application. Instead, based on the transitivity of total neighbourhoods, using closure under consistency proves to be a more natural choice.

\subsection{Density}

The notion of continuity that we employ in our setting implies that if we are given an estimate $V$ on a value $f(x)$, then we can find an adequate estimate $U_{V}$ on the argument $x$ of $f$; let us highlight this elementary fact since we will need it later on.

Lemma 5.1 (Finite support). Let $f: \rho \rightarrow \sigma$ and $x: \rho$. For every $V \in \operatorname{Con}_{\sigma}$ with $V \subseteq f(x)$, there exists a $U_{V} \in \operatorname{Con}_{\rho}$ such that $U_{V} \subseteq x$ and $\left\langle U_{V}, V\right\rangle \subseteq f$.

Proof. From (FS) it follows directly that if $b \in f(x)$, then there exists a $U_{b} \subseteq x$ such that $\left\langle U_{b}, b\right\rangle \in f$ due to the deductive closure of $f$. Assuming then that $V$ is such that $V \subseteq f(x)$, for $U_{V}:=\bigcup_{b \in V} U_{b}$, we indeed have $U_{V} \subseteq x$, and also $\left\langle U_{V}, V\right\rangle \subseteq f$ by the deductive closure of $f$.

An ideal $x: \rho$ is a total ideal, for which we write $G_{\rho}(x)$ or $x \in G_{\rho}$, if it conforms to the following inductive clauses:

$$
\begin{aligned}
G_{l}(x) & :=\underset{P \in \operatorname{Con}_{l}^{g}}{\exists} P \subseteq x, \\
G_{\rho \rightarrow \sigma}(f) & :=\underset{x: \rho}{\forall}\left(G_{\rho}(x) \rightarrow G_{\sigma}(f x)\right) .
\end{aligned}
$$

Note that the base-type definition is equivalent to demanding the existence of a $p \in \operatorname{Tok}_{l}^{g}$ such that $p \in x$. Totality of ideals is upwards closed.

Lemma 5.2 (Extension lemma). At type $\rho$, if $x, y: \rho$ are such that $G_{\rho}(x)$ and $x \subseteq y$, then $G_{\rho}(y)$.

Proof. At a base type $l$, let $G_{l}(x)$ and $y: l$ be two ideals with $x \subseteq y$. Then, there is a total token $p \in \operatorname{Tok}_{l}^{g}$, such that $p \in x$, so also $p \in y$. At a higher type $\rho \rightarrow \sigma$, let $G_{\rho \rightarrow \sigma}(f), g: \rho \rightarrow \sigma$, and assume that $f \subseteq g$. We want to show that $g$ is also total, so consider an arbitrary $x$ with $G_{\rho}(x)$. By the totality of $f$, we have that $G_{\sigma}(f x)$, and since it is straightforward to see that $f x \subseteq g x$, we get $G_{\sigma}(g x)$ by the induction hypothesis at $\sigma$.

The main argument starts with the following obvious observation.

Lemma 5.3. At every type, if a neighbourhood is transitive, then its consistency closure is an ideal (and the converse holds as well). 
Proof. Let $\rho$ be a type and $U \in \operatorname{Con}_{\rho}^{\text {ctr }}$. By transitivity, every two tokens in the consistency closure of $U$ will be consistent, and the consistency closure is already deductively closed: $U^{\prime} \subseteq \widetilde{U}$ means $U \asymp_{\rho} U^{\prime}$ by definition, so if $U^{\prime} \vdash_{\rho} a$, then propagation yields $U \asymp_{\rho} a$, hence, $a \in \widetilde{U}$ as well. The converse is also direct to show.

By Theorem 4.9, an immediate consequence of this lemma is that the consistency closure of a total neighbourhood is an ideal, so it suffices to show that, for a given $P \in \mathrm{Con}_{\rho}^{g}$, we must have $G_{\rho}(\widetilde{P})$. Consider the following statements for an arbitrary type $\rho$.

$$
\begin{aligned}
& \underset{\Gamma \in \operatorname{Fin}_{\rho}^{o}}{\forall} \underset{x \in G_{\rho}}{\forall} \underset{U^{x} \in \operatorname{Con}_{\Gamma}^{\mathrm{ctr}}}{\exists} U^{x} \asymp_{\rho} x, \\
& \underset{U, U^{\prime} \in \operatorname{Con}_{\rho}}{\forall} \underset{x \in G_{\rho}}{\forall}\left(U^{\prime} \star_{\rho} U \asymp_{\rho} x \rightarrow \underset{U_{0} \in \operatorname{Con}_{\rho}}{\exists}\left(U \vdash_{\rho} U_{0} \subseteq x \wedge U^{\prime} \star_{\rho} U_{0}\right)\right), \\
& \underset{P \in \operatorname{Con}_{\rho}^{g}}{\forall} \widetilde{P} \in G_{\rho}, \\
& \underset{U \in \operatorname{Con}_{\rho}}{\forall} \underset{x \in G_{\rho}}{\exists} U \subseteq x .
\end{aligned}
$$

The first one is an expression of infinitary omniception, as it states that an omniceptive finite set accepts each total ideal by being consistent with it with one of its transitive neighbourhoods. The second expresses inconsistency preserving witnessing of the consistency between a total ideal and a neighbourhood; the claimed witness is stronger than the neighbourhood itself, since it lies below both the total ideal and the neighbourhood, and in a sense to be made clearer after Lemma 5.8 below, it provides the missing feature from omniception that we need to achieve totality on the level of ideals. The third one is the crux of our strategy, as it says that the consistency closure of a total neighbourhood is a total ideal, and the fourth one, of course, is density.

Proposition 5.4 (Conditional density). Let $\rho$ be a type. If (C) holds in $\rho$, then also (D) holds in $\rho$.

Proof. Let $U$ be any neighbourhood at type $\rho$. By Theorem 4.7, there exists a total neighbourhood $P^{U}$ such that $U \asymp{ }_{\rho} P^{U}$. Then, $U \subseteq \widetilde{P^{U}}$ by definition, whereas $\widetilde{P^{U}} \in G_{\rho}$ by $(\mathrm{C})$. We set $x:=\widetilde{P^{U}}$ and we are done.

Lemma 5.5. Every base type satisfies (O), (W), (C) and (D).

Proof. Let $\imath$ be some base type. To show (O), consider an omniceptive finite set $\Gamma$ and a total ideal $x$. By the totality of $x$, there is some $P \in \operatorname{Con}_{l}^{g}$ such that $P \subseteq x$, and by the omniception of $\Gamma$ there is some $U^{P} \in \operatorname{Con}_{\Gamma}^{\text {ctr }}$ such that $U^{P} \asymp{ }_{1} P$. Set $U^{x}:=U^{P}$. Then, for every $U \subseteq x$, we have $U^{x} \asymp_{l} P \asymp_{l} U$, which implies $U^{x} \asymp_{l} U$ by the total transitivity of $l$ (Proposition 4.3), so $U^{x} \asymp, x$.

\footnotetext{
$\|$ Notice that, here, we only needed weak omniception from $\Gamma$. Furthermore, observe that in the flat setting this argument would fail due to the requirement of finiteness of $\Gamma$.
} 
To show (W), let $U$ and $U^{\prime}$ be neighbourhoods and $x$ be a total ideal, such that $U^{\prime} \Varangle_{l} U \asymp_{l} x$. By the totality of $x$, there exists a total neighbourhood $P$ such that $P \subseteq x$. We have of course $P \asymp{ }_{1} U$, which, since total tokens are maximal at base types, implies that $P \vdash_{l} U$. This in turn implies that $U \subseteq x$ by the deductive closure of $x$, so we may set $U_{0}:=U$, which trivially meets the stated requirements.

To show (C), let $P$ be some total neighbourhood. Then, there is some total token $p \in \mathrm{Tok}_{l}$ with $P \vdash p$; a fortiori we have $P \asymp \asymp_{l} p$, so $p \in \widetilde{P}$ by the definition of consistency closure. Since by Lemma 5.3 , the set $\widetilde{P}$ is an ideal, we conclude that it is in fact total.

Finally, that every base type is dense, we get from Proposition 5.4, since (C) already holds.

Proposition 5.6 (Omniception). Let $\rho$ and $\sigma$ be types. If (C) holds in $\rho$ and (O) holds in $\sigma$, then $(\mathrm{O})$ holds in $\rho \rightarrow \sigma$.

Proof. Let $\Theta \in \operatorname{Fin}_{\rho \rightarrow \sigma}^{o}$ and $f \in G_{\rho \rightarrow \sigma}$. By the finite omniception of $\Theta$, we know that each $U \in \operatorname{Con}_{L(\Theta)}^{\max }$ is a total neighbourhood, so by (C) at $\rho$, we have $\widetilde{U} \in G_{\rho}$. By the totality of $f$, we have that $f(\widetilde{U}) \in G_{\sigma}$, so there will be some $V^{f(\widetilde{U})} \in \operatorname{Con}_{\Theta \cdot U}^{\mathrm{ctr}}$ such that $V^{f(\widetilde{U})} \asymp_{\sigma} f(\widetilde{U})$, because $\Theta \cdot U$ is omniceptive by the finite omniception of $\Theta$ and $(\mathrm{O})$ at $\sigma$. Based on these, we may set

$$
W^{f}:=\bigcup_{U \in \operatorname{Con}_{L(\Theta)}^{\max }}\left\langle U, V^{f(\widetilde{U})}\right\rangle .
$$

We have $W^{f} \in \operatorname{Con}_{\Theta}^{\text {ctr }}$ by Lemma 3.12 (2). Furthermore, let $\left\langle U_{0}, b_{0}\right\rangle \in W$ and $\langle U, b\rangle \in f$ be such that $U \asymp \asymp_{\rho} U_{0}$; then $U \subseteq \widetilde{U_{0}}$ (remember that $U_{0}$ is a total neighbourhood) and consequently, $\bar{U} \subseteq \widetilde{U_{0}}$ by the propagation of consistency; by the monotonicity of $f$, we get $f(\bar{U}) \subseteq f\left(\widetilde{U_{0}}\right)$, so since $b \in f(\bar{U})$ it must also be $b \in f\left(\widetilde{U_{0}}\right)$; but $f\left(\widetilde{U_{0}}\right) \asymp_{\sigma} V^{f\left(\widetilde{U_{0}}\right)}$ and $b_{0} \in V^{f\left(\widetilde{U_{0}}\right)}$, so $b \asymp{ }_{\sigma} b_{0}$, as we wanted.

Proposition 5.7 (Witnessing). Let $\rho$ and $\sigma$ be types. If (D) holds in $\rho$ and (W) holds in $\sigma$, then (W) holds in $\rho \rightarrow \sigma$.

Proof. Let $f \in G_{\rho \rightarrow \sigma}$ and $W, W^{\prime} \in \operatorname{Con}_{\rho \rightarrow \sigma}$ be such that $W^{\prime} \Varangle_{\rho \rightarrow \sigma} W \asymp_{\rho \rightarrow \sigma} f$. For $i=1, \ldots, m$, let $U_{i}^{\prime} \in \operatorname{Con}_{L\left(W^{\prime}\right)}$ and $U_{i} \in \operatorname{Con}_{L(W)}$ run through all witnessing pairs of inconsistency between $W^{\prime}$ and $W$, that is, cover all the cases where

$$
U_{i}^{\prime} \asymp_{\rho} U_{i} \wedge W^{\prime} \cdot U_{i}^{\prime} \star_{\sigma} W \cdot U_{i}
$$

By (D) at $\rho$, for each $i$ there exists an $x_{i} \in G_{\rho}$ such that $U_{i}^{\prime} \cup U_{i} \subseteq x_{i}$. By the consistency of (upper) application, for every such $x_{i}$, we have $W^{\cdot} U_{i} \asymp_{\sigma} f\left(x_{i}\right)$, and by (W) at $\sigma$ there exists some $V_{i 0} \in \mathrm{Con}_{\sigma}$ such that $W \cdot U_{i} \vdash_{\sigma} V_{i 0} \subseteq f\left(x_{i}\right)$ and $W^{\prime} \cdot U_{i}^{\prime} \Varangle_{\sigma} V_{i 0}$. By Lemma 5.1, there exists some $U_{V_{i 0}} \subseteq x_{i}$ for every $i$ such that $\left\langle U_{V_{i 0}}, V_{i 0}\right\rangle \subseteq f$. Letting $U_{i 0}:=U_{V_{i 0}} \cup U_{i}^{\prime} \cup U_{i}$, by the deductive closure of $f$, it follows that $\left\langle U_{i 0}, V_{i 0}\right\rangle \subseteq f$. Since by the hypotheses at $\sigma$, for every $i$, we have

$$
\left\langle U_{i}, W \cdot U_{i}\right\rangle \vdash_{\rho \rightarrow \sigma}\left\langle U_{i 0}, V_{i 0}\right\rangle \subseteq f \wedge\left\langle U_{i}^{\prime}, W^{\prime} \cdot U_{i}^{\prime}\right\rangle \Varangle_{\rho \rightarrow \sigma}\left\langle U_{i 0}, V_{i 0}\right\rangle,
$$


it follows that

$$
W \vdash_{\rho \rightarrow \sigma} \bigcup_{i=1}^{m}\left\langle U_{i 0}, V_{i 0}\right\rangle \subseteq f \wedge W^{\prime} \Varangle_{\rho \rightarrow \sigma} \bigcup_{i=1}^{m}\left\langle U_{i 0}, V_{i 0}\right\rangle,
$$

so, we may set $W_{0}:=\bigcup_{i=1}^{m}\left\langle U_{i 0}, V_{i 0}\right\rangle$ and we are done.

We may generalise the property (W) to account for inconsistency preserving witnesses of the consistencies between a total ideal and neighbourhoods in a finite set.

Lemma 5.8. At a type $\rho$, the statement (W) is equivalent to the following: Let $\Gamma \in \operatorname{Fin}_{\rho}$ and $x \in G_{\rho}$; for all $U \in \operatorname{Con}_{\Gamma}$ with $U \asymp_{\rho} x$, there exists a neighbourhood $N_{U, \Gamma, x} \in \operatorname{Con}_{\rho}$ such that

$$
U \vdash_{\rho} N_{U, \Gamma, x} \subseteq x \wedge \underset{U^{\prime} \in \operatorname{Con}_{\Gamma}}{\forall}\left(U^{\prime} \star_{\rho} U \rightarrow U^{\prime} \star_{\rho} N_{U, \Gamma, x}\right) .
$$

Proof. Let $\Gamma$ be a finite set, $U$ some neighbourhood of $\Gamma$ and $x$ a total ideal. Assume that (W) holds, and furthermore that $U_{1}, \ldots, U_{m} \in \operatorname{Con}_{\Gamma}$ are all neighbourhoods in $\Gamma$ such that $U_{i} \star_{\rho} U$ for $i=1, \ldots, m$. Then, for each such $i$ there is a neighbourhood $U_{0 i} \in \operatorname{Con}_{\rho}$ such that $U_{i} \Varangle_{\rho} U_{0 i}$ and $U \vdash_{\rho} U_{0 i} \subseteq x$. Setting $N_{U, \Gamma, x}:=\bigcup_{i=1}^{m} U_{0 i}$, we are done. In the other way around, let $U$ and $U^{\prime}$ be two neighbourhoods and $x$ a total ideal, such that $U^{\prime} \Varangle_{\rho} U \asymp_{\rho} x$, and assume that (W') holds for all finite sets $\Gamma$, neighbourhoods $U \subseteq \Gamma$ and total ideals $x$. Setting $U_{0}:=N_{U, U \cup U^{\prime}, x}$, we are done.

So, if $\Gamma$ accepts a total ideal $x$ at all, even if with a nontransitive neighbourhood $U$, then it could be safely side extended to include a common part $N_{U, \Gamma, x}$ of $\bar{U}$ and $x$; enriched in this way $\Gamma$ would now accept $x$ in the strong sense of inclusion. This is exactly what we need to exploit by taking the consistency closure of a higher-type total neighbourhood, provided its list of arguments is omniceptive. But let us get to the details without further ado.

Proposition 5.9 (Closure). Let $\rho$ and $\sigma$ be types. If (O) and (W) hold in $\rho$ and (C) holds in $\sigma$, then (C) holds in $\rho \rightarrow \sigma$.

Proof. Let $T \in \operatorname{Con}_{\rho \rightarrow \sigma}^{g}$ and $x \in G_{\rho}$. We show that $\widetilde{T}(x) \in G_{\sigma}$. Based on Lemma 3.9, we may assume that $L(T) \in \operatorname{Fin}_{\rho}^{o}$ without harming generality. By $(\mathrm{O})$ at $\rho$, there exists a $U^{x} \in \operatorname{Con}_{L(T)}^{\mathrm{ctr}}$ such that $U^{x} \asymp_{\rho} x$. By Lemma 4.8 (4), we have $T \cdot U^{x} \in \operatorname{Con}_{\sigma}^{g}$, and by (C) at $\sigma$, we have $\widetilde{T \cdot U^{x}} \in G_{\sigma}$. So, in order to show that $\widetilde{T}(x) \in G_{\sigma}$, it suffices to show that $\widetilde{T \cdot U^{x}} \subseteq \widetilde{T}(x)$ and invoke Lemma 5.2.

Let then $b \in \operatorname{Tok}_{\sigma}$ be such that $b \in \widetilde{T \cdot U^{x}}$. This means that $b \asymp_{\sigma} T \cdot U^{x}$. By Lemma 3.7 (2), we have $\left\langle U^{x}, b\right\rangle \asymp_{\rho \rightarrow \sigma} T$. By (W) at $\rho$ and Lemma 5.8, there exists a neighbourhood $U_{0}^{x}:=N_{U^{x}, L(T), x} \in \operatorname{Con}_{\rho}$ such that

$$
U^{x} \vdash_{\rho} U_{0}^{x} \subseteq x \wedge \underset{U^{\prime} \in \operatorname{Con}_{L(T)}}{\forall}\left(U^{\prime} \star_{\rho} U^{x} \rightarrow U^{\prime} \star_{\rho} U_{0}^{x}\right) ;
$$

we have $\left\langle U_{0}^{x}, b\right\rangle \asymp_{\rho \rightarrow \sigma} T$, because for every $\left\langle U^{\prime}, b^{\prime}\right\rangle \in T$ with $U^{\prime} \asymp_{\rho} U_{0}^{x}$ it has to be $U^{\prime} \asymp_{\rho} U^{x}$ from the above, therefore, $b \asymp_{\sigma} b^{\prime}$ follows by $\left\langle U^{x}, b\right\rangle \asymp_{\rho \rightarrow \sigma} T$. We have found a $U_{b}:=U_{0}^{x} \in \operatorname{Con}_{\rho}$ such that $\left\langle U_{b}, b\right\rangle \asymp_{\rho \rightarrow \sigma} T$ and $U_{b} \subseteq x$; but this means by definition that $b \in \widetilde{T}(x)$, and we are done. 
Theorem 5.10 (Density). Every type satisfies $(\mathrm{O}),(\mathrm{W})$ and $(\mathrm{C})$, and in particular, every type is dense.

Proof. It follows by a mutual induction over types by Lemma 5.5 and Propositions 5.4, 5.6, 5.7 and 5.9.

As a closing remark, we should note that the witness that we provide is actually the maximal total extension of a given neighbourhood, in the sense that if, for a type $\rho$, $U \in \operatorname{Con}_{\rho}$ is some neighbourhood, $U^{g} \in \operatorname{Con}_{\rho}^{g}$ is the witness provided by Theorem 4.7, and $x \in G_{\rho}$ is such that $U \subseteq x$, then $x \subseteq \widetilde{U^{g}}$.

\subsection{Nontotality of transitive ideals}

In the same way as we did with finite totality and transitivity in Theorem 4.9, we would like to know if we can connect totality and transitivity on the level of ideals, and possibly obtain an explicit characterisation of totality in terms of consistency. We show that this is not possible.

In Berger $(1999,2002)$, an element $x$ is defined to be almost maximal when $y_{1} \supseteq x \subseteq y_{2}$ implies $y_{1} \asymp y_{2}$ for all $y_{1}$ and $y_{2}$. At the same time, we call $x$ transitive if $y_{1} \asymp x \asymp y_{2}$ implies $y_{1} \asymp y_{2}$ for all $y_{1}, y_{2}$. We also extend the bar notation for the deductive closure to ideals, in particular, we write $\overline{x \cup y}$ to mean the set $\left\{a \mid \exists_{U \subseteq x \cup y} U \vdash a\right\}$. We immediately see the following.

Lemma 5.11. At every type, an ideal is almost maximal if and only if it is transitive.

Proof. That transitivity implies almost maximality is clear. To see the converse, let $x$ be almost maximal and $y_{1} \asymp x \asymp y_{2}$. Then, $x \subseteq \overline{y_{i} \cup x}$ for each $i$ and we get $\overline{y_{1} \cup x} \asymp \overline{y_{2} \cup x}$ by almost maximality, which yields $y_{1} \asymp y_{2}$.

It is well known that in hierarchies over flat base types there exist functionals that are maximal - and therefore, by the above lemma, transitive - but not total, typical examples being various second-order minimization operators (Stoltenberg-Hansen et al. 1994, Example 8.3.2, Exercise 8.5.14). In the nonflat setting, we have counterexamples already at inductive base types, as we will see immediately. For the following, we express the transitivity of $x$ through tokens, similarly to Lemma 3.4.

Proposition 5.12 (Total-transitivity). At any type, total ideals are transitive. Conversely, there exist types with transitive ideals that are not total.

Proof. At a base type $l$, let $a_{1}, a_{2}$ be tokens and $x$ a total ideal, such that $a_{1} \asymp_{1} x \asymp_{1} a_{2}$. There exists a total neighbourhood $P$ with $P \subseteq x$, so the assumption yields $a_{1} \asymp{ }_{1} P \asymp_{1} a_{2}$, which implies $a_{1} \asymp_{l} a_{2}$ by the finite total transitivity of $l$ (Proposition 4.3).

At a higher type $\rho \rightarrow \sigma$, let $\left\langle U_{1}, b_{1}\right\rangle,\left\langle U_{2}, b_{2}\right\rangle$ be tokens and $f$ be a total ideal, such that $\left\langle U_{1}, b_{1}\right\rangle \asymp_{\rho \rightarrow \sigma} f \asymp_{\rho \rightarrow \sigma}\left\langle U_{2}, b_{2}\right\rangle$. Assume furthermore that $U_{1} \asymp_{\rho} U_{2}$. By Theorem 5.10, there exists a total ideal $x: \rho$ such that $U_{1} \cup U_{2} \subseteq x$. Since $f$ is itself total, the ideal $f(x): \sigma$ must also be total, and by the induction hypothesis at $\sigma$ it must also be transitive. 
Now, applying all terms of the assumption to $x$, we obtain $b_{1} \asymp{ }_{\sigma} f(x) \asymp{ }_{\sigma} b_{2}$, which then yields $b_{1} \asymp{ }_{\sigma} b_{2}$.

For the converse, consider the ideal $\infty=\left\{\mathbf{S}^{m} * \mid m \geqslant 0\right\}$ of type $\mathbb{N}$.

\subsection{Noncontinuity of totalisation}

The witness for density that we have provided in Section 5.1 is a mapping of the sort tot : $\operatorname{Con}_{\rho} \rightarrow \rho .^{\dagger \dagger}$ It is easy to see that this is not a 'continuous' mapping - that is, it does not extend to an ideal of type $\rho \rightarrow \rho$ - since it can not be expected to preserve consistency: consider the neighbourhoods $\left\{\mathrm{S}^{*}\right\}$ and $\left\{\mathrm{SS}^{*}\right\}$ at type $\mathbb{N}$; these are consistent with each other, but

$$
\operatorname{tot}(\{\mathrm{S} *\}) \ni \mathrm{S} 0 \neq_{\mathbb{N}} \mathrm{SS} 0 \in \operatorname{tot}(\{\mathrm{SS} *\}) .
$$

This counterexample is general enough to convince us that this shortcoming is not particular to our witness.

Lemma 5.13. There is no consistency-preserving mapping $t: \operatorname{Con}_{\mathbb{N}} \rightarrow \mathbb{N}$ such that $U \subseteq t(U)$ and $t(U) \in G_{\mathbb{N}}$ for all $U \in \operatorname{Con}_{\mathbb{N}}$.

Proof. If such a mapping existed, we would have $t\left(U_{1}\right) \asymp \mathbb{N} t\left(U_{2}\right)$ for any two neighbourhoods $U_{1}, U_{2} \subseteq \infty$. Fixing such a $U_{1}$ with $t\left(U_{1}\right)=\overline{\left\{\mathrm{S}^{n} 0\right\}}$ for some $n$ and setting $U_{2}:=\left\{\mathrm{S}^{n+1} *\right\}$, we get $t\left(U_{1}\right) \varlimsup_{\mathbb{N}} t\left(U_{1}\right)$, a contradiction.

\subsection{Separation}

One of Berger's key insights in Berger (1990), which permeates all subsequent approaches that our work is based upon (including our own) was that the notion of totality can be clarified if density is viewed together with an accompanying notion of 'separation': intuitively, a type $\rho$ is considered to feature the separation property, if any two open sets of conflicting information can be told apart by a total 'predicate' of type $\rho \rightarrow \mathbb{B}$. His argument proceeded by mutual induction for both properties of density and separation over all finite types. What we did instead in our mutual inductive arguments above was in effect to replace the notion of 'separation of neighbourhoods by infinite total ideals' by notions of 'acceptance of total ideals by finite sets.' In our exposition, separation follows as a simple corollary of density.

Following Schwichtenberg et al. (2012), and assuming the presence of the booleans in the type system, call a type $\rho$ separating if

$$
\underset{U, U^{\prime} \in \mathrm{Con}_{\rho}}{\forall}\left(U \star_{\rho} U^{\prime} \rightarrow \underset{f \in G_{\rho \rightarrow \mathbb{B}}}{\exists}\langle U, \mathrm{tt}\rangle \in f \ni\left\langle U^{\prime}, \text { ff }\right\rangle\right),
$$

and finitely separating if

$$
\underset{U, U^{\prime} \in \operatorname{Con}_{\rho}}{\forall}\left(U \Varangle_{\rho} U^{\prime} \rightarrow \underset{T \in \operatorname{Con}_{\rho \rightarrow \mathbb{B}}^{g}}{\exists}\langle U, \mathrm{tt}\rangle \asymp_{\rho \rightarrow \mathbb{B}} T \asymp_{\rho \rightarrow \mathbb{B}}\left\langle U^{\prime}, \text { ff }\right\rangle\right) .
$$

\footnotetext{
$\dagger \dagger$ Such mixed typings of terms appear often and naturally in considerations within information systems, and should be accounted for in a theory of partial computable functionals together with their approximations as in Huber et al. (2010).
} 
Proposition 5.14 (Separation). Every type is finitely separating, and consequently separating.

Proof. If $U$ and $U^{\prime}$ are inconsistent a $\rho$, then the finite set $\left\{\langle U, \mathrm{tt}\rangle,\left\langle U^{\prime}, \mathrm{ff}\right\rangle\right\}$ is a neighbourhood at $\rho \rightarrow \mathbb{B}$, and by Theorem 4.7, there will exist some $T \in \operatorname{Con}_{\rho \rightarrow \mathbb{B}}^{g}$ which side extends it. Consequently, by Theorem 5.10, the total ideal $\widetilde{T}$ will extend it.

\section{Notes}

We gave a new, bottom-up proof of the Kreisel density theorem for finite types interpreted over nonflat inductive base types given as algebras by constructors. We introduced a notion of totality for neighbourhoods and proved a finite density theorem, which states that, given a neighbourhood, one may first totalise it in an explicitly finitary way to obtain a total neighbourhood. Kreisel density is obtained by extending this total neighbourhood to a total ideal by means of consistency; the resulting ideal, though generated by a compact element, is the maximal totalisation of the given neighbourhood. Here, we gather notes on the above, on related literature, and on future work.

The density theorem in the literature. As already pointed out Section 1, the density problem was addressed for the first time by Kreisel (1959) and also Kleene (1959). In his Ph.D. thesis (Berger 1990), he recast and solved the density problem within domain theory, generalising results of Ershov (1975b, 1977) and paralleling work of Normann (1989) - see Berger (1993) and Stoltenberg-Hansen et al. (1994) for an account in English. A proof which does not thematise separation is given by Normann (1999), while a modern approach from a viewpoint of an all-encompassing theory of higher-type computability can be found in the recent volume by Longley and Normann (2015). The density theorem is a fundamental result with several deep and far-reaching applications, like the choice theorem (Kreisel 1959; Berger 1993; Schwichtenberg 1996), Kreisel's representation theorem (Kreisel 1959; Normann 1981, 1997), a generalised Kreisel-Lacombe-Shoenfield theorem (Berger 1993), Normann's theorem (Normann 2000a,b; Plotkin 1999), and Escardó's theory of exhaustive search (Escardó 2007, 2008), as well as extensions and generalisations, for example, to dependent and universe domains (Berger 1999b), to Scott's equilogical spaces (Bauer and Birkedal 2000), or even to an account of totality independently of density (Normann 1996) - see also, Berger (1999, 2002), Normann (1999, 2008). It would be natural to seek among these studies for ones that would benefit from the possibility of explicitly finitary totalisation. Existence of such cases would further justify the extension of the results presented here to richer type systems, starting with the one adopted in Schwichtenberg et al. (2012), and possibly moving on to the type systems covered in Berger (1999b).

Related work. The problem of finding a proof of density theorem 'by compacts' occurred to the author back in the early 2011, and, since then, tackling it has primarily provided an incentive to develop the theory of nonflat information systems for semantics (see, Karádais 2018, for examples of collateral results). A partial result in the direction of finite witnesses 
for density was presented in Karádais (2013), where, in contrast to the present approach, it was shown that one may first prove a version of finite separation at every type and then use this as a lemma to prove density (a version of our Proposition 5.7 also appears there); that approach provided a satisfactory finitary explanation of separation but not of totalisation. Meanwhile, an alternative bottom-up approach to the density theorem, which grew independently but turned out to be similar in spirit to ours, was carried out by Rinaldi (2014). Rinaldi offers a nonflat semantics that is topological rather than domaintheoretic: he uses certain formal topologies (Sambin 1987), for which he proves that they are equivalent to unary information systems; these are information systems where in addition neighbourhoods always have eigentokens, that is, for every $U \in$ Con, there exists some $a \in$ Tok such that $U \sim\{a\}$. In our setting this is true of base types, but not of higher types. To adapt Rinaldi's semantics in a way that clearly matches broader categories of information systems than just the unary ones, and look at a formal-topological proof of density by compacts anew, would not only be instructive, but it could also provide a more succinct and elegant proof.

Towards a common study of totality and cototality. Recently, 'cototal ideals,' that is, total ideals together with infinities like $\infty$ at type $\mathbb{N}$, have been used to model stream-like objects at base types arising from initial algebras, offering an alternative to versions of semantics simultaneously based on initial algebras and final coalgebras (Rutten 2000; Hancock et al. 2009; Ghani et al. 2009); for this line of work, rooted in Berger (2009, 2011) and Berger and Seisenberger (2012), see Berger et al. (2011, 2016), Schwichtenberg et al. (2012), Miyamoto et al. (2013) and Miyamoto and Schwichtenberg (2015). In view of the mismatch between transitivity and totality in a nonflat setting, which we described in Section 5.2, it looks like a refinement is possible, where totality should feature an increased degree of finiteness and should be studied hand in hand with an appropriate notion of cototality: beside more or less obvious differences of the two at base types (based on the proof of Lemma 5.13, for example, one could expect continuous 'cototalisations' to exist), their interplay at higher types remains terra incognita at the time of this writing.

I thank Matthias Hofer for the feedback, Davide Rinaldi for the stimulating exchange of information, Parménides García Cornejo and Kenji Miyamoto for hearing me out, Apostolos Damialis for his advice on the notation and Dirk-André Deckert for all the backup. Special thanks to the referees for their substantial feedback: they shook me out of some misconceptions, posed insightful questions and helped make the text a little more readable.

\section{References}

Bauer, A. and Birkedal, L. (2000). Continuous functionals of dependent types and equilogical spaces. In: Proceedings of the 14th EASCL Annual Conference on Computer Science Logic (CSL '2000), Fischbachau, Germany, August 21-26, Springer, 202-216.

Berger, U. (1990). Totale Objekte und Mengen in der Bereichstheorie (Total objects and sets in domain theory). Univ. München, Fak. für Mathematik. ii, $122 \mathrm{~S}$. 
Berger, U. (1993). Total sets and objects in domain theory. Annals of Pure and Applied Logic 60 (2) 91-117.

Berger, U. (1999a). Continuous functionals of dependent and transfinite types. In: Models and Computability. Invited Papers from the Logic Colloquium '97, European Meeting of the Association for Symbolic Logic, Leeds, UK, July 6-13, Cambridge University Press, 1-22.

Berger, U. (1999b). Effectivity and density in domains: A survey. In: A Tutorial Workshop on Realizability Semantics and Applications. A Workshop Associated to the Federated Logic Conference, Trento, Italy, June 30-July 1, Elsevier, 13.

Berger, U. (2002). Computability and totality in domains. Mathematical Structures in Computer Science 12 (3) 281-294.

Berger, U. (2009). Realisability and adequacy for (co)induction. In: Proceedings of the 6th International Conference on Computability and Complexity in Analysis (CCA'09), August 18-22, Ljubljana, Slovenia, Schloss Dagstuhl - Leibniz Zentrum für Informatik, 12.

Berger, U. (2011). From coinductive proofs to exact real arithmetic: Theory and applications. Logical Methods in Computer Science 7 (1) 24.

Berger, U., Miyamoto, K., Schwichtenberg, H. and Seisenberger, M. (2011). Minlog-A tool for program extraction supporting algebras and coalgebras. In: Algebra and Coalgebra in Computer Science - Proceedings of the 4th International Conference (CALCO '11), Winchester, UK, August 30-September 2, 393-399.

Berger, U., Miyamoto, K., Schwichtenberg, H. and Tsuiki, H. (2016). Logic for gray-code computation. In Dieter, P. and Peter, S. (eds.): Concepts of Proof in Mathematics, Philosophy, and Computer Science. De Gruyter.

Berger, U. and Seisenberger, M. (2012). Proofs, programs, processes. Theory of Computing Systems 51 (3) 313-329.

Ershov, Y.L. (1975a). Maximal and everywhere-defined functionals. Algebra Logic 13 210-225.

Ershov, Y.L. (1975b). Theorie der Numerierungen. II (Theory of numberings. II) Übersetzung aus dem Russischen: H.-D. Hecker. Wissenschaftliche Redaktion: G. Asser. Berlin: VEB Deutscher Verlag der Wissenschaften. M 15.00 (1976). Sonderdruck aus Z. math. Logik Grundl. Math. 21 473-584 (1975).

Ershov, Y.L. (1977). Model $\mathbb{C}$ of partial continuous functionals. In: Logic Colloquium 76, Proceedings of a Conference held at Oxford in 1976, Studies in Logic and the Foundations of Mathematics, vol. 87, 455-467.

Escardó, M.H. (2007). Infinite sets that admit fast exhaustive search. In: Proceedings of the 22nd Annual IEEE Symposium on Logic in Computer Science (LICS '07), Washington, DC, USA, IEEE Computer Society, 443-452.

Escardó, M.H. (2008). Exhaustible sets in higher-type computation. Logical Methods in Computer Science 4 (3) 37.

Ghani, N., Hancock, P.G. and Pattinson, D. (2009). Continuous functions on final coalgebras. In: Proceedings of the 25th Conference on the Mathematical Foundations of Programming Semantics (MFPS '09), Oxford, UK, April 3-7, Elsevier, 3-18.

Hancock, P.G., Ghani, N. and Pattinson, D. (2009). Representations of stream processors using nested fixed points. Logical Methods in Computer Science 5 (3) 17.

Huber, S. (2010). On the computational content of choice axioms. Master's thesis, Mathematisches Institut, LMU.

Huber, S., Karádais, B.A. and Schwichtenberg, H. (2010). Towards a formal theory of computability. In: Ways of Proof Theory (Pohler's Festschrift), Ontos Verlag, 257-282.

Karádais, B.A. (2013). Towards an Arithmetic with Approximations. Ph.D. thesis, Mathematisches Institut, LMU. 
Karádais, B.A. (2016). Atomicity, coherence of information, and point-free structures. Annals of Pure and Applied Logic 167 (9) 753-769.

Karádais, B.A. (2018). Normal forms, linearity, and prime algebraicity over nonflat domains. Mathematical Logic Quarterly, to appear.

Kleene, S.C. (1959). Countable functionals. In: Heyting, A. (ed.) Constructivity in Mathematics: Proceedings of the Colloquium held at Amsterdam in 1957, Studies in Logic and the Foundations of Mathematics. North-Holland Publishing Co, 81-100.

Kreisel, G. (1959). Interpretation of analysis by means of constructive functionals of finite types. In: A. Heyting (eds.) Constructivity in Mathematics: Proceedings of the Colloquium held at Amsterdam, 1957, Studies in Logic and the Foundations of Mathematics. North-Holland Publishing Co, 101128.

Longley, J. and Normann, D. (2015). Higher-Order Computability, Springer.

Miyamoto, K., Forsberg, F.N. and Schwichtenberg, H. (2013). Program extraction from nested definitions. In: Interactive Theorem Proving - Proceedings of the 4th International Conference (ITP '13), Rennes, France, July 22-26, 370-385.

Miyamoto, K. and Schwichtenberg, H. (2015). Program extraction in exact real arithmetic. Mathematical Structures in Computer Science 25 (8) 1692-1704.

Normann, D. (1981). Countable functionals and the projective hierarchy. Journal of Symbolic Logic 46 209-215.

Normann, D. (1989). Kleene-spaces. In: Logic Colloqium '88, Proceedings of the Colloqium held at Padova/Italy in 1988, Studies in Logic and the Foundations of Mathematics, vol. 127 91-109.

Normann, D. (1996). A hierarchy of domains with totality, but without density. In: Computability, Enumerability, Unsolvability. Directions in Recursion Theory, Cambridge University Press, 233-257.

Normann, D. (1997). Closing the gap between the continuous functionals and recursion in ${ }^{3} E$. Archive for Mathematical Logic 36 (4-5) 269-287.

Normann, D. (1999). The continuous functionals. In: Handbook of Computability Theory, Elsevier, 251-275.

Normann, D. (2000a). Computability over the partial continuous functionals. Journal of Symbolic Logic 65 (3) 1133-1142.

Normann, D. (2000b). The Cook-Berger problem. A guide to the solution. In: Proceedings of the Domains IVth Workshop, Haus Humboldtstein, Remagen-Rolandseck, Germany, October 2-4, Elsevier, 9.

Normann, D. (2009). Applications of the Kleene-Kreisel density theorem to theoretical computer science. In: New Computational Paradigms. Changing Conceptions of What is Computable, Springer, $119-138$.

Plotkin, G.D. (1978). $T^{\omega}$ as a universal domain. Journal of Computer and System Sciences 17 (2) 209-236.

Plotkin, G.D. (1999). Full abstraction, totality and PCF. Mathematical Structures in Computer Science 9 (1) $1-20$.

Rinaldi, D. (2014). Formal methods in the theories of rings and domains. Ph.D. thesis, Mathematisches Institut, LMU.

Rutten, J.J.M.M. (2000). Universal coalgebra: A theory of systems. Theoretical Computer Science 249 (1) 3-80.

Sambin, G. (1987). Intuitionistic formal spaces--A first communication. In Skordev, D. G. (ed.): Mathematical Logic and its Applications (Druzhba, 1986), Plenum, 187-204.

Schwichtenberg, H. (1996). Density and choice for total continuous functionals. In Odifreddi, P. (ed.): Kreiseliana: About and Around Georg Kreisel, A K Peters, 335-362. 
Schwichtenberg, H. (2007). Recursion on the partial continuous functionals. In: Dimitracopoulos, C., Newelski, L., Normann, D. and Steel, J. (eds.), Logic Colloquium ('05), Lecture Notes in Logic, vol. 28, Association for Symbolic Logic, 173-201.

Schwichtenberg, H. and Wainer, S.S. (2012). Proofs and Computations, Perspectives in Logic, Cambridge University Press.

Scott, D.S. (1982). Domains for denotational semantics. In: Automata, Languages and Programming (Aarhus, 1982), Lecture Notes in Computer Science, vol. 140, Springer, 577-613.

Stoltenberg-Hansen, V., Lindström, I. and Griffor, E.R. (1994). Mathematical Theory of Domains, Cambridge Tracts in Theoretical Computer Science, vol. 22, Cambridge University Press.

Winskel, G. and Larsen, K.G. (1984). Using information systems to solve recursive domain equations effectively. In Kahn, G., MacQueen, D. B., Plotkin G. D. (eds.): Semantics of Data Types, SpringerVerlag, 109-129. 